\title{
LILRB1 polymorphisms influence posttransplant HCMV susceptibility and ligand interactions
}

\author{
Kang Yu, ${ }^{1}$ Chelsea L. Davidson, ${ }^{1}$ Agnieszka Wójtowicz, ${ }^{2}$ Luiz Lisboa, ${ }^{1,3}$ Ting Wang, ${ }^{1}$ Adriana M. Airo, ${ }^{1}$ Jean Villard, ${ }^{4}$ \\ Jeremie Buratto, ${ }^{5}$ Tatyana Sandalova, ${ }^{5}$ Adnane Achour, ${ }^{5}$ Atul Humar, ${ }^{6}$ Katia Boggian, ${ }^{7}$ Alexia Cusini, ${ }^{8}$ Christian van Delden, ${ }^{9}$ \\ Adrian Egli, ${ }^{10,11}$ Oriol Manuel, ${ }^{12}$ Nicolas Mueller, ${ }^{13}$ Pierre-Yves Bochud, ${ }^{2}$ Swiss Transplant Cohort Study, ${ }^{14}$ and Deborah N. Burshtyn ${ }^{1}$ \\ ${ }^{1}$ Medical Microbiology and Immunology, University of Alberta, Edmonton, Alberta, Canada. ${ }^{2}$ Infectious Diseases Service, University Hospital and University of Lausanne, Lausanne, Switzerland. ${ }^{3}$ Department \\ of Medicine, University of Alberta, Edmonton, Alberta, Canada. ${ }^{4}$ Immunology and Transplant Unit, Service of Nephrology, Geneva University Hospital, Geneva, Switzerland. ${ }^{5}$ Science for Life Laboratory, \\ Department of Medicine Solna, Karolinska Institute, and Department of Infectious Diseases, Karolinska University Hospital, Solna, Stockholm, Sweden. ${ }^{6}$ Multi-Organ Transplant Program, University of \\ Toronto, Toronto, Ontario, Canada. 'Division of Infectious Diseases and Hospital Epidemiology, Cantonal Hospital St. Gallen, St. Gallen, Switzerland. ${ }^{8}$ Department of Infectious Diseases and Hospital \\ Epidemiology, Bern University Hospital and University of Bern, Bern, Switzerland. ${ }^{9}$ Transplant Infectious Diseases Unit, University Hospitals Geneva, Geneva, Switzerland. ${ }^{10}$ Division of Clinical Microbiology, \\ University Hospital Basel, Basel, Switzerland. "Applied Microbiology Research, Department of Biomedicine, University of Basel, Basel, Switzerland. ${ }^{12}$ Infectious Diseases Service and Transplantation Center, \\ University Hospital and University of Lausanne, Lausanne, Switzerland. ${ }^{13}$ Division of Infectious Diseases and Hospital Epidemiology, University Hospital Zurich, University of Zurich, Zurich, Switzerland. \\ ${ }^{14}$ The Swiss Transplant Cohort Study is detailed in the Supplemental Acknowledgments
}

\begin{abstract}
UL18 is a human CMV (HCMV) MHC class I (MHCI) homolog that efficiently inhibits leukocyte immunoglobulin-like receptor subfamily B member 1 (LILRB1)+ NK cells. We found an association of LILRB1 polymorphisms in the regulatory regions and ligand-binding domains with control of HCMV in transplant patients. Naturally occurring LILRB1 variants expressed in model NK cells showed functional differences with UL18 and classical MHCI, but not with HLA-C. The altered functional recognition was recapitulated in binding assays with the binding domains of LILRB1. Each of 4 nonsynonymous substitutions in the first 2 LILRB1 immunoglobulin domains contributed to binding with UL18, classical MHCI, and HLA-C. One of the polymorphisms controlled addition of an $\mathrm{N}$-linked glycan, and that mutation of the glycosylation site altered binding to all ligands tested, including enhancing binding to UL18. Together, these findings indicate that specific LILRB1 alleles that allow for superior immune evasion by HCMV are restricted by mutations that limit LILRB1 expression selectively on NK cells. The polymorphisms also maintained an appropriate interaction with HLA-C, fitting with a principal role of LILRB1 in fetal tolerance.
\end{abstract}

\section{Introduction}

NK cells play a critical role during the early stages of human CMV (HCMV) infection, and the presence of HCMV alters the NK compartment $(1,2)$. The alterations include expansion of $\mathrm{NKG} 2 \mathrm{C}^{+}$cells, changes in expression of signaling molecules leading to enhanced antibody-dependent cell-mediated cytotoxicity (ADCC), and epigenetic changes that regulate cytokine production (reviewed in refs. 3, 4). HCMV has several known strategies to evade the NK response, such as disrupting the expression of ligands for the activating receptor NKG2D by UL16, enhancing HLA-E expression via a UL40-derived peptide to inhibit NK cells via NKG2A, and expression of the MHCI mimic UL18 (5). NK cells are inhibited by UL18 through engagement of the inhibitory receptor leukocyte immunoglobulin-like receptor subfamily B member 1 (LILRB1) $(6,7)$. UL18 shares about 25\% sequence similarity with classical MHCI molecules (8), binds cellular peptides efficiently within its binding groove, and associates with $\beta_{2} \mathrm{~m}$. The bulk of the molecule is shielded from the adaptive immune system by extensive glycosylation that is noticeably absent from the contact region

Conflict of interest: The authors have declared that no conflict of interest exists

Submitted: July 12, 2017; Accepted: January 25, 2018

Reference information: / Clin Invest. 2018;128(4):1523-1537.

https://doi.org/10.1172/JCI96174. with LILRB1 $(8,9)$. UL18 binds to the 2 membrane-distal immunoglobulin domains of LILRB1 $(6,10)$ in a manner that parallels the interaction of LILRB1 with MHCI (11). However, the interface with UL18 is substantially larger compared with MHCI, with enhanced complementarity, explaining the higher affinity of LILRB1 to UL18 compared with MHCI (11).

Several observations suggest that the interaction of LILRB1 with UL18 is important in the immune response to HCMV. In transplant patients, higher expression of LILRB1 occurs on lymphocytes prior to the onset of viremia $(12,13)$. LILRB1 is expressed on NK cells that have expanded in response to acute HCMV infection (14). In addition, mutations in the $\alpha 1$ and $\alpha 3$ domains of UL18 among HCMV strains impacts interaction with LILRB1 (15). A recent study indicates that variation in UL18 sequence does impact LILRB1 ${ }^{+} \mathrm{NK}$ cell control of HCMV replication in vitro (16).

LILRB1 exhibits considerable diversity in the population, and polymorphisms in the LILRB1 gene have been associated with susceptibility to rheumatoid arthritis (17) and weakly associated with HCMV disease in a subset of patients with HIV (18). However, to our knowledge, no studies to date have examined how LILRB1 polymorphisms influence the interaction of LILRB1 with UL18. LILRB1 is expressed by a variety of immune cells including monocytes, DCs, B cells, subsets of effector and memory $\mathrm{T}$ 
A

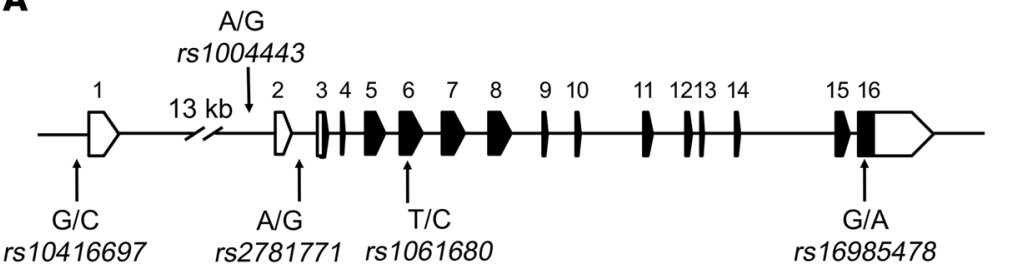

B

rs10416697

(Distal promoter)
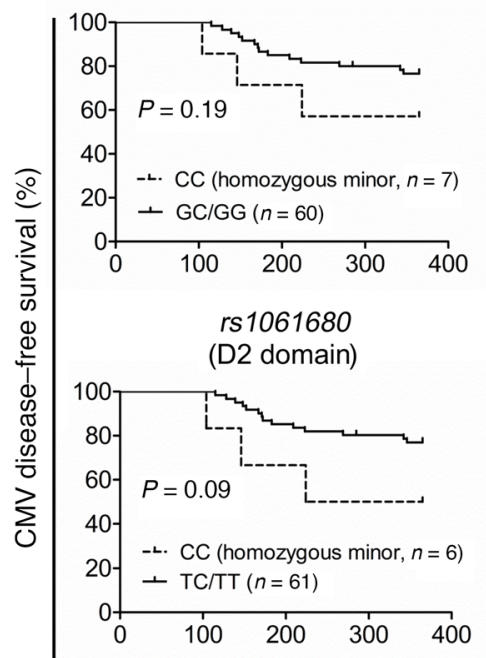

Days from transplant

E

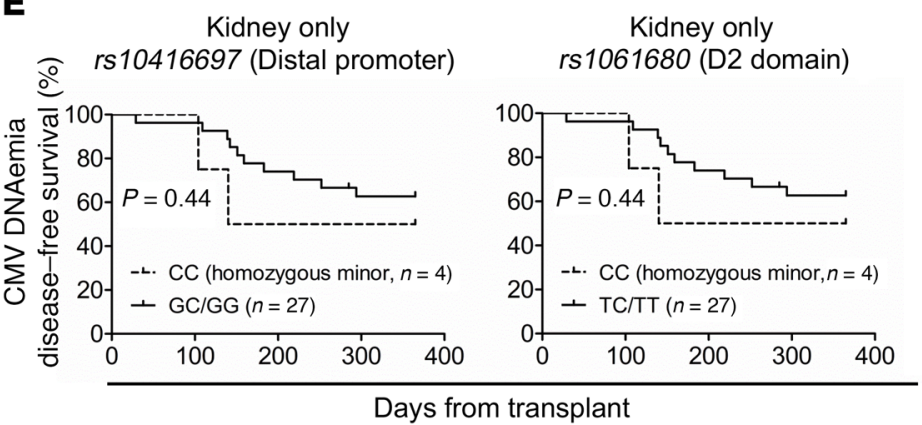

C

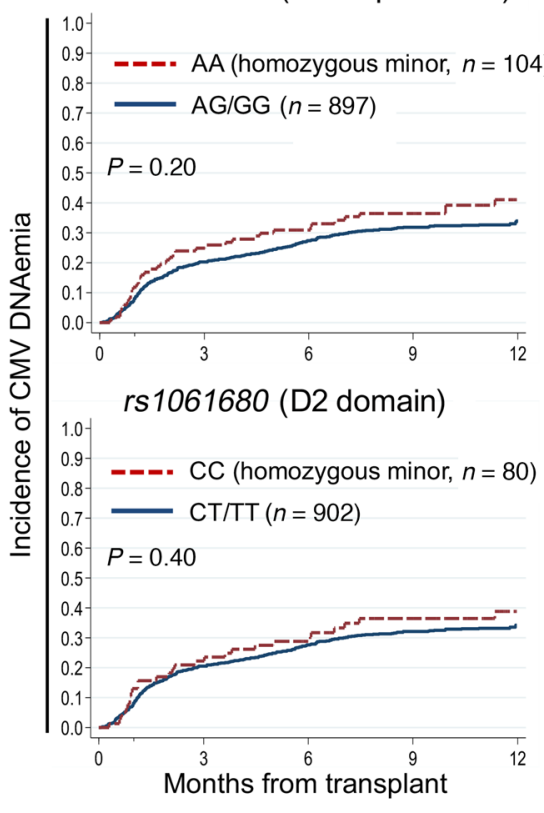

D

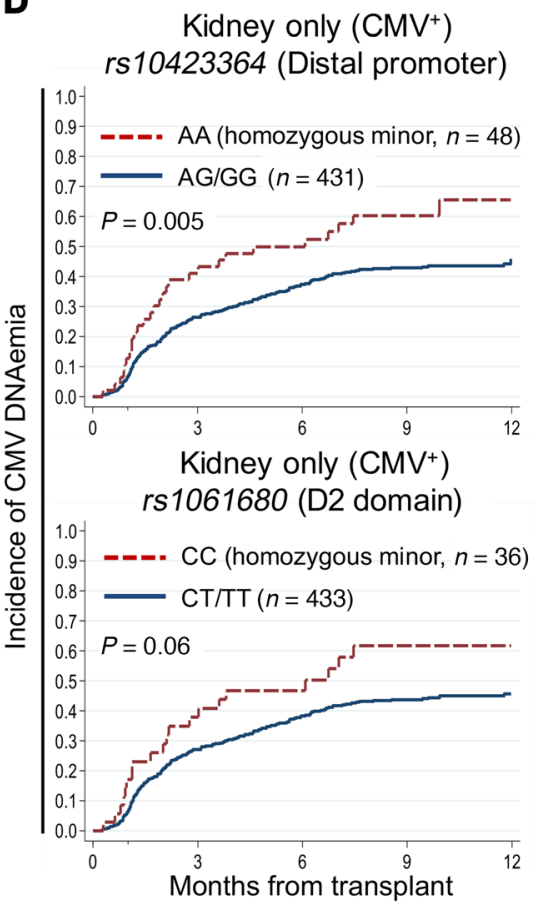

Figure 1. LILRB1 genotype and control of HCMV replication in transplant patients. (A) Schematic illustrating the positions of 5 SNPs analyzed in transplant patients. (B) Disease-free survival rates for HCMV disease of $67 \mathrm{D}+/ \mathrm{R}$ - Canadian transplant patients genotyped for the indicated SNPs. The $P$ values indicated in each graph in Figure 1 were determined by log-rank (Mantel-Cox) test. (C) Incidence of HCMV DNAemia for $r s 10423364$ ( $n=762$ ) and $r s 1061680$ $(n=748)$ of all the SOT patients from the STCS. (D) Incidence of posttransplant HCMV DNAemia of D+/R- or R+ STCS kidney transplant patients for the indicated SNPs $(n=479)$. (E) Kaplan-Meier curves of HCMV DNAemia disease-free status according to alleles of rs10423364 and rs1061680 in 31 Canadian kidney transplant patients.

cells, and $20 \%-70 \%$ of NK cells $(6,19,20)$. We previously defined several haplotypes of the LILRB1 gene and their relationship to LILRB1 expression on NK cells in healthy individuals (7, 17, 21). More specifically, individuals with the SNPs $r s 1004443-\mathrm{A}$, rs3760860-G, and rs3760861-G have higher levels of LILRB1 transcript and surface expression on NK cells (7) than those with the SNPs rs1004443-G, rs3760860-A, and rs3760861-A. Given the ability of UL18 to inhibit NK cell responses and the apparent importance of NK cells in controlling HCMV replication, we investigated LILRB1 polymorphisms in transplant patients to test the hypothesis that individuals with greater LILRB1 expression on NK cells would exhibit poorer control of HCMV replication. In con- 
trast to our expectations, the results revealed that the emergence of alleles conferring lower frequency of LILRB1 ${ }^{+} \mathrm{NK}$ cells may be an adaptation to limit the expression of receptor variants that are more susceptible to manipulation by UL18. In spite of the adaptation, individuals with lower frequency of LILRB1 ${ }^{+} \mathrm{NK}$ cells remain disadvantaged in controlling HCMV under immunosuppression.

\section{Results}

LILRB1 polymorphisms and HCMV viremia in transplant patients. To test whether LILRB1 genotype influences HCMV susceptibility, we analyzed LILRB1 genotypes in a small group of Canadian transplant patients (22). The number of samples available for this retrospective analysis was only 67 , but all patients enrolled in the study were HCMV seronegative prior to receiving an organ from an HCMV-positive donor and therefore predisposed to high rates of primary HCMV disease (Supplemental Table 1; supplemental material available online with this article; https://doi.org/10.1172/ JCI96174DS1). All patients received antiviral prophylaxis for 3-6 months to prevent HCMV primary infection and were followed for 1 year after transplantation for incidence of HCMV asymptomatic infection (50\%) and disease (25\%). We analyzed 5 SNPs spread throughout the LILRB1 gene that encompass the known regulatory regions, a nonsynonymous change in binding domain, and the cytoplasmic region of the protein (Figure 1A). We used rs10416697 (-14895 from the translational start in the distal promoter region) and rs1004443 (-1026), which form extended haplotypes in the regulatory domains (Supplemental Figure 1A). The third SNP ( $r$ 2781771) is at -225 relative to the start codon and the minor allele is quite rare. The fourth SNP ( $r$ 1061680) causes a nonsynonymous substitution in the second immunoglobulin domain of the receptor previously examined in the context of HIV and HCMV (18). The final and most 3' SNP tested is at position +5724 relative to the translational start ( $r$ 16985478), with a second nonsynonymous change in the cytoplasmic tail that encodes a potential ubiquitination site (7). Given the limited sample size, only trends could be ascertained. Counter to our prediction that patients with more LILRB1 ${ }^{+}$NK cells would have a higher incidence of HCMV disease, we found that homozygous carriers of the minor allele at $r s 10416697$ were more prone to presenting with HCMV disease $(50 \%$ in $\mathrm{C} / \mathrm{C}$ vs. $23 \%$ in $\mathrm{T} / \mathrm{C}+\mathrm{T} / \mathrm{T}$; Fisher's exact test, $P=0.166$; log-rank test, $P=0.0873$ ) (Figure $1 \mathrm{~B}$ and Supplemental Figure 2). Results for $r s 1004443$ are identical to $r s 10416697$ (data not shown) and there is a slightly more pronounced trend for rs1061680. There was no substantial difference in the incidence of HCMV disease in patients with different genotypes for $r s 2781771$ or rs16985478 (Figure 1B).

We next examined a larger cohort to validate the putative association of LILRB1 SNP rs1061680 and HCMV asymptomatic infection and/or disease using patients from the Swiss Transplant Cohort Study (STCS) (23). Genotype data for both $r s 1061680$ and its potential surrogate upstream SNP $r s 10423364\left(r^{2} \sim 0.8\right)$ were available for 1,018 STCS solid organ transplant (SOT) recipients of European descent, of which $76 \%$ had donor and/or recipient HCMV-positive serostatus (Supplemental Table 2). Of the 776 seropositive patients, 651 had received kidneys, and of those kidney recipients, $74 \%$ had donor and/or recipient HCMV-positive serostatus. An additional 100 patients had genotype data for rs1061680 but not for $r s 10423364$. The SNP rs10423364 is almost in perfect linkage disequilibrium (LD) with SNP rs10416697 $\left(r^{2} \sim 0.96\right)$ in the distal promoter and in perfect LD with the proximal promoter SNP $r s 1004443\left(r^{2}=1\right)$.

In univariate analysis, there was no association between LILRB1 SNPs and virus replication within the entire STCS population (Figure 1C). However, when the analyses were restricted to kidney transplant recipients (Supplemental Figure 3), we detected a significant association with $r s 10423364$ (log-rank test, $P=0.02$ ) and a trend for rs1061680 $(P=0.25)$ with HCMV infection. These associations were even more significant when analyses were further restricted to HCMV-positive patients $(P=0.005$ and $P=0.06$, respectively; Figure 1D). We found a trend toward higher viremia only in the kidney transplant patients in the Canadian cohort. There were just 31 patients in total, so significance is unclear (Figure $1 \mathrm{E}$ and Supplemental Figure 2). There was no association with HCMV disease (Supplemental Figure 3) in the whole population or within a subset of kidney recipients only. The rates of disease were much lower in the STCS cohort, presumably due to the highly selected D+/R- set of the Canadian samples. In order to determine whether the association with HCMV disease was independent from other covariates, we analyzed the role of rs1061680 and rs10423364 in multivariate models. We first included SNP rs10423364 in the model, since we observed that it had the strongest association with HCMV disease (Supplemental Table 3). The association was still significant in the multivariate model (HR $=1.69 ; 95 \%$ CI, 1.13-2.54; $P=0.01)$ after adjustment for donor and recipient age and sex, use of corticosteroid, cyclosporine and tacrolimus regimen, rejection episodes, use of antiviral prophylaxis, and HCMV serostatus. We included both SNPs in the multivariate stepwise model, but only rs10423364 remained independently associated with HCMV. No significant association was observed with killer cell immunoglobulin-like receptor (KIR) haplotypes that were further entered in the model, perhaps due to the smaller sample size or due to association of the KIR locus, which is also encoded within the leukocyte receptor complex located on chromosome 19. There was no significant association with HCMV when using rs1061680 instead of $r s 10423364$, either with or without adjustment for KIR variables $(\mathrm{HR}=1.46 ; 95 \% \mathrm{CI} 0.92-2.32 ; P=0.1)$. Altogether, these data reveal a possible role for a genetic variation in LILRB1 in the control of HCMV replication in STCS cohort kidney transplant patients. The stronger association with $r s 10423364$ than with $r$ 1061680 in the larger cohort may be due to the most important SNP(s) being located in the interval between these 2SNPs. The interval between $r s 1004443$ and rs1061680 contains several SNPs, including 2 additional nonsynonymous changes in the first immunoglobulin domain. The 2 SNPs located in the gene regulatory region are in perfect LD $\left(r^{2}\right.$ =1) and share the same LD value with $r s 1061680\left(r^{2}=0.76\right)$ (Table 1). Among the other 3 known nonsynonymous SNPs in the binding domain (D1D2) coding region of LILRB1, rs1061679 (L/P) is also in strong LD with the 3 SNPs that we found were associated with HCMV infection $\left(r^{2}=0.92\right)$, but $r s 12460501(\mathrm{~A} / \mathrm{T})$ and $r s 1061681(\mathrm{~S} / \mathrm{I})$ are not (Table 1). Based on previous studies of LILRB1 polymorphisms by our laboratory and others $(7,17)$ and data drawn from the 1000 Genomes Project (24), there is 1 major haplotype and several minor LILRB1 haplotypes that combine the 4 nonsynonymous SNPs located in the D1D2 coding region, as shown in Figure 2. According to the published $3 \mathrm{D}$ structures, none of the residues affected by the polymorphisms 
Table 1. LD acquired from the 1000 Genomes Project phase 1 on European populations

$\begin{array}{lccccccc}\text { LD }\left(r^{2}\right) & \text { rs10416697 } & \text { rs10423364 } & \text { rs1004443 } & \text { rs1061679 } & \text { rs12460501 } & \text { rs1061680 } & \text { rs1061681 } \\ \text { rs10416697 } & - & 0.98 & 0.98 & 0.91 & 0.22 & 0.76 & 0.50 \\ r \text { r10423364 } & & - & 1.00 & 0.92 & 0.22 & 0.76 & 0.50 \\ \text { rs1004443 } & & & - & 0.92 & 0.22 & 0.76 & 0.50 \\ \text { rs1061679 } & & & & - & 0.24 & 0.68 & 0.53 \\ \text { rs12460501 } & & & & & - & 0.29 & 0.28 \\ \text { rs1061680 } & & & & & & - & 0.65 \\ \text { rs1061681 } & & & & & & & -\end{array}$

Bold indicates SNPs analyzed in transplant patients.

lead to major changes in the conformation of the receptor. We confirmed the function of the receptors in YTS cells using 221 cells expressing HLA-G and the antibody W6/32 to block the recognition of HLA-G and prevent the inhibition of YTS cells (Supplemental Figure 5). Lysis of 221 cells by YTS-LAIS and YTSPTTI was similarly and reproducibly reduced compared with parental YTS cells (Figure 3D, top panel), but the difference did not reach a measurable level of significance in

make direct contact with UL18 or HLA-A2, although they do all align on the same face of the receptor. The residues proline/leucine and threonine/alanine corresponding to rs1061679 and rs12460501, respectively, are located near the interface, whereas residues threonine/isoleucine and isoleucine/serine corresponding to rs1061680 and rs1061681, respectively, are localized quite far away (Supplemental Figure 4A). Furthermore, the leucine to proline change can have substantial impact on protein structure and conformational dynamics because proline introduces rigidity into the backbone and leucine is a larger hydrophobic residue.

LILRB1 variants differ for functional inhibition of NK cells. To test potential functional differences between the LILRB1 variants, we established an in vitro system with conventional 721.221 tumor targets expressing surface UL18. The 721.221 cell line was transduced with retroviral vector encoding UL18 with HA tag at the N-terminus and yellow fluorescent protein (YFP) at the Cterminus. UL18 expression levels are shown as measured with anti-HA and the intrinsic YFP expression relative to the parental cell line (Figure 3A).

To directly compare the function of the 2 LILRB1 variants that differ at all 4 polymorphic positions in D1D2, we expressed the 2 variants denoted as LAIS and PTTI in LILRB1-negative NK cells. We transduced YTS cells with HA-tagged LAIS-LILRB1 and HA-tagged PTTI-LILRB1, and selected subclones with similar surface expression levels of LILRB1 by flow cytometry using histone PARylation factor 1 (HPF1) and $\alpha$-HA (Figure 3B). The similar ratio of HA to HPF1 suggests that the 4 amino acid differences do not

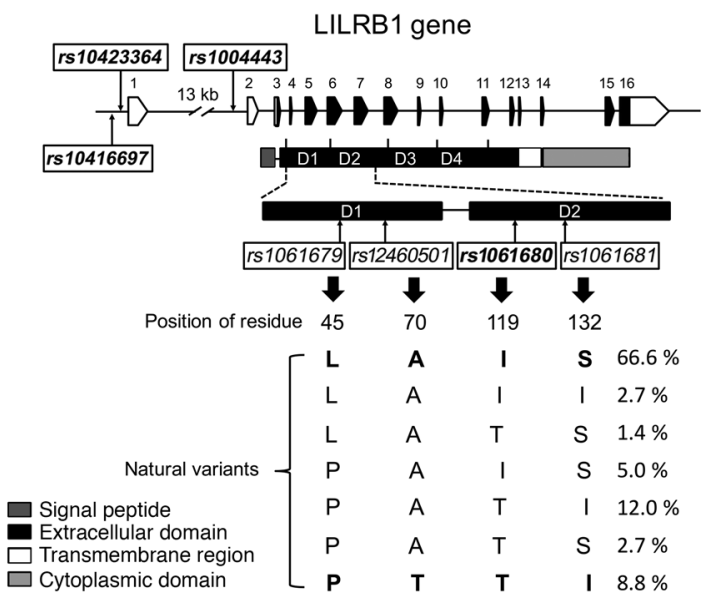

aggregated data (Figure 3D, bottom panel). The lower lysis of the MHCI-deficient target is likely due to the expression of LILRB1 tempering the signaling in YTS cells in a cell-intrinsic manner. The lysis of 221 cells bearing HLA-Cw15 or UL18 was lower for YTS-PTTI (Figure 3C) compared with YTS-LAIS (Figure 3D), suggesting a better interaction by PTTI. There was no significant difference between the 2 LILRB1 variants with the more potent ligand HLA-G (Figure 3D, lower panel). Treatment with HPF1 antibody to block LILRB1 function increases the lysis of Cw15, UL18, and HLA-G target cells to the same level as YTS cells, demonstrating that receptor interaction with the ligand is indeed inhibiting the YTS cells (Figure 3E).

LILRB1 binding with HLA-I molecules and viral UL18. To probe the relationship of polymorphisms with the binding properties of the variants, we generated Fc-tagged versions of the D1 and D2 domains of the 2 LILRB1 variants. The 2 variants bind equally well to the antibody HPF1 as previously reported (17). The LAIS variant migrated slightly faster than the PTTI variant by SDS-PAGE analysis (Figure 4). We compared binding of the $\mathrm{Fc}$ fusion protein to 221 cells expressing MHCI ligands over a range of concentrations at $4^{\circ} \mathrm{C}$ as detected by flow cytometry. The raw binding data are illustrated in Supplemental Figure 6. The signal was higher at all concentrations for the PTTI-Fc variant compared with the LAIS-Fc variant with HLA-Cw15 and HLA-B58 (Figure 4C). Consistent with the functional assays (Figure 3), binding of the 2 variants to cells expressing HLA-G was similar. The binding was not saturated, which precludes formal comparison of halfmaximal binding concentrations. The binding specificity was demonstrated by blocking with the antibody W6/32 (Figure 4D). We next compared the binding of the LILRB1 variants to 221 cells expressing UL18. We observed lower binding of LAIS-Fc compared with PTTI-Fc (Figure 4E), although the difference in binding to UL18 was less pronounced than with HLA-Cw15 and HLA-B58. We performed a reciprocal binding assay using purified UL18 Fc fusion protein and HA-tagged full-length LILRB1PTTI and -LAIS expressed on RBL cells normalized to the receptor levels using $\alpha-\mathrm{HA}$ and HPF1, respectively (Figure $4 \mathrm{~F}$ ). Again,

Figure 2. Natural protein variants deduced from the 1000 Genomes Project for the first $\mathbf{2}$ Ig domains of LILRB1. The location of the 4 nonsynonymous SNPs and corresponding amino acids are indicated with the haplotype proportions shown on the right. Bold indicates the LILRB1 variants tested in the functional assay. 
A
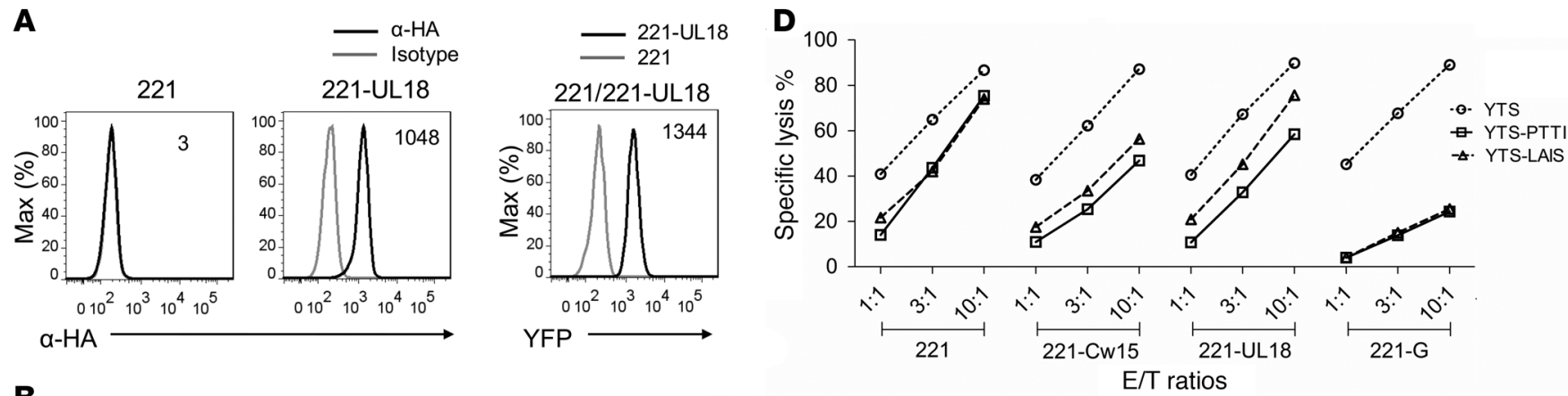

B
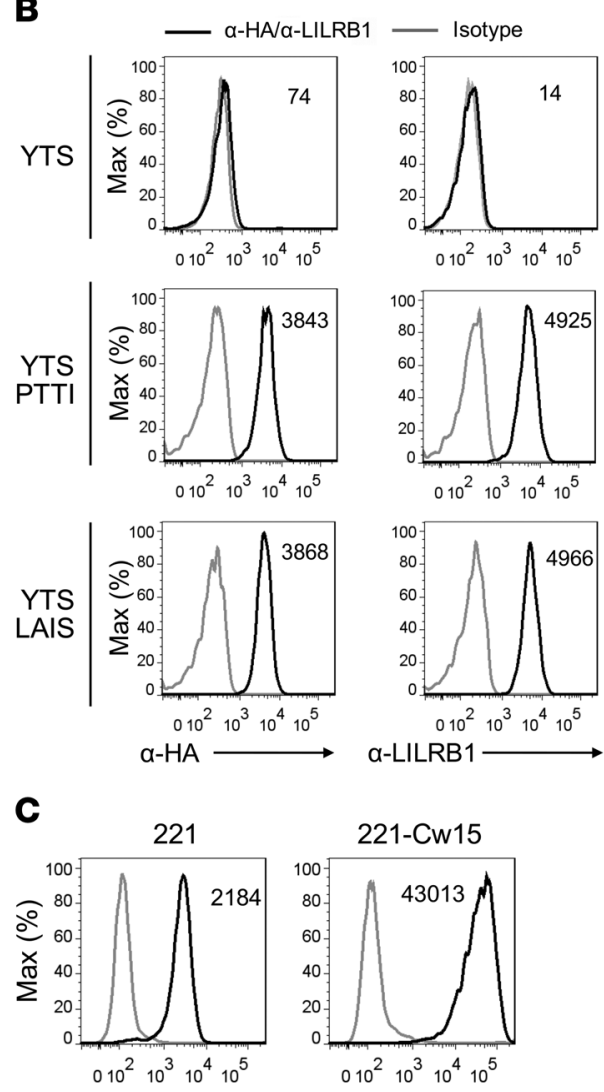

E
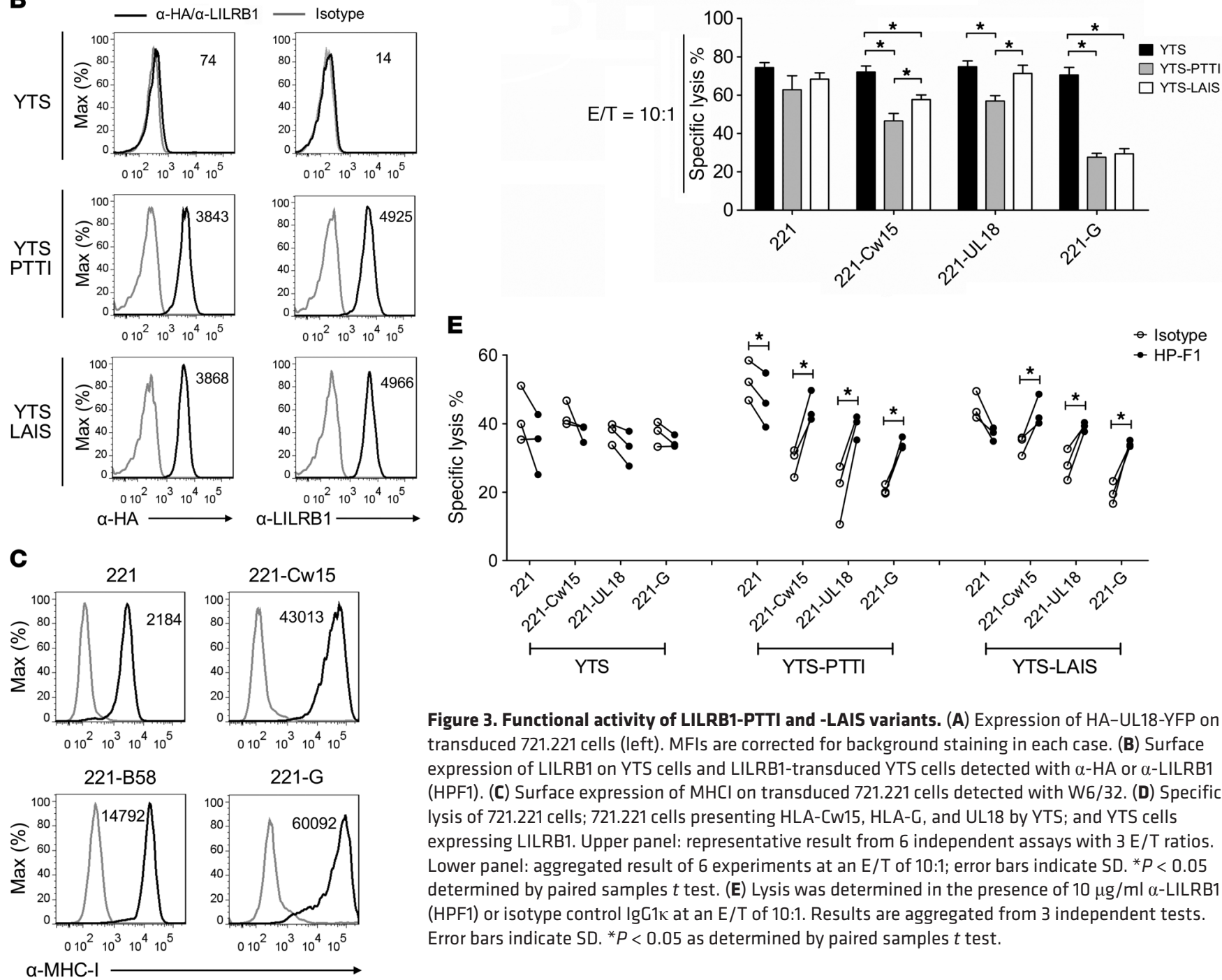

Figure 3. Functional activity of LILRB1-PTTI and -LAIS variants. (A) Expression of HA-UL18-YFP on transduced 721.221 cells (left). MFIs are corrected for background staining in each case. (B) Surface expression of LILRB1 on YTS cells and LILRB1-transduced YTS cells detected with $\alpha$-HA or $\alpha$-LILRB1 (HPF1). (C) Surface expression of MHCI on transduced 721.221 cells detected with W6/32. (D) Specific lysis of 721.221 cells; 721.221 cells presenting HLA-Cw15, HLA-G, and UL18 by YTS; and YTS cells expressing LILRB1. Upper panel: representative result from 6 independent assays with $3 \mathrm{E} / \mathrm{T}$ ratios. Lower panel: aggregated result of 6 experiments at an E/T of 10:1; error bars indicate SD. ${ }^{*} P<0.05$ determined by paired samples $t$ test. (E) Lysis was determined in the presence of $10 \mu \mathrm{g} / \mathrm{ml} \alpha$-LILRB1 (HPF1) or isotype control lgG1 $\kappa$ at an E/T of 10:1. Results are aggregated from 3 independent tests. Error bars indicate SD. ${ }^{*} P<0.05$ as determined by paired samples $t$ test.

LILRB1-PTTI bound better to UL18-Fc than LILRB1-LAIS most clearly at the highest concentration tested (900 nM), although the assay was limited by the amount of UL18-Fc available (Figure 4G).

The results of the binding assays are consistent with the differences observed in the functional assays, although it is unclear why differences in binding with MHCI were not observed by Kuroki et al. (17). Residue N117 is predicted to be glycosylated in LILRB1PTTI but not LILRB1-LAIS due to the presence of a threonine residue at position 119 (ref. 17 and Figure 5A). An additional N-linked carbohydrate in PTTI at residue 117 would also explain the difference in migration that we observed between the LAIS and PTTI variants on SDS-PAGE (Figure 4A), and a finding that the additional carbohydrate was important for binding would explain the discrepancy with the previous report due to their production of the receptors in E. coli.

Residue $T 119$ is required for glycosylation of N117, and it differentially influences ligand binding. To test whether glycosylation of PTTI was responsible for the difference in migration, we treated the purified $\mathrm{Fc}$ fusion proteins with $\mathrm{N}$-glycosidase. Treatment with $\mathrm{N}$-glycosidase under denaturing conditions resulted in the 2 LILRB1 variants running at a similar size (Figure $5 \mathrm{~B}$ ). To investigate whether the N-linked glycosylation plays a role in LILRB1-PTTI binding, 

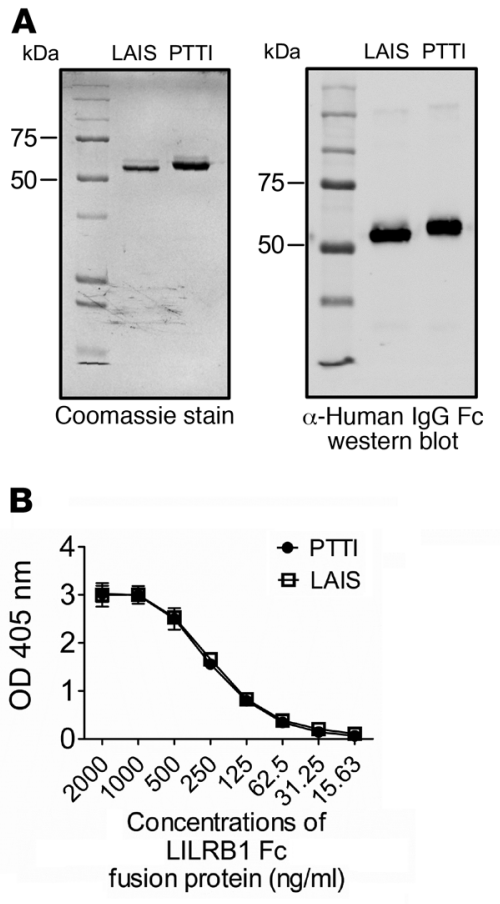

C
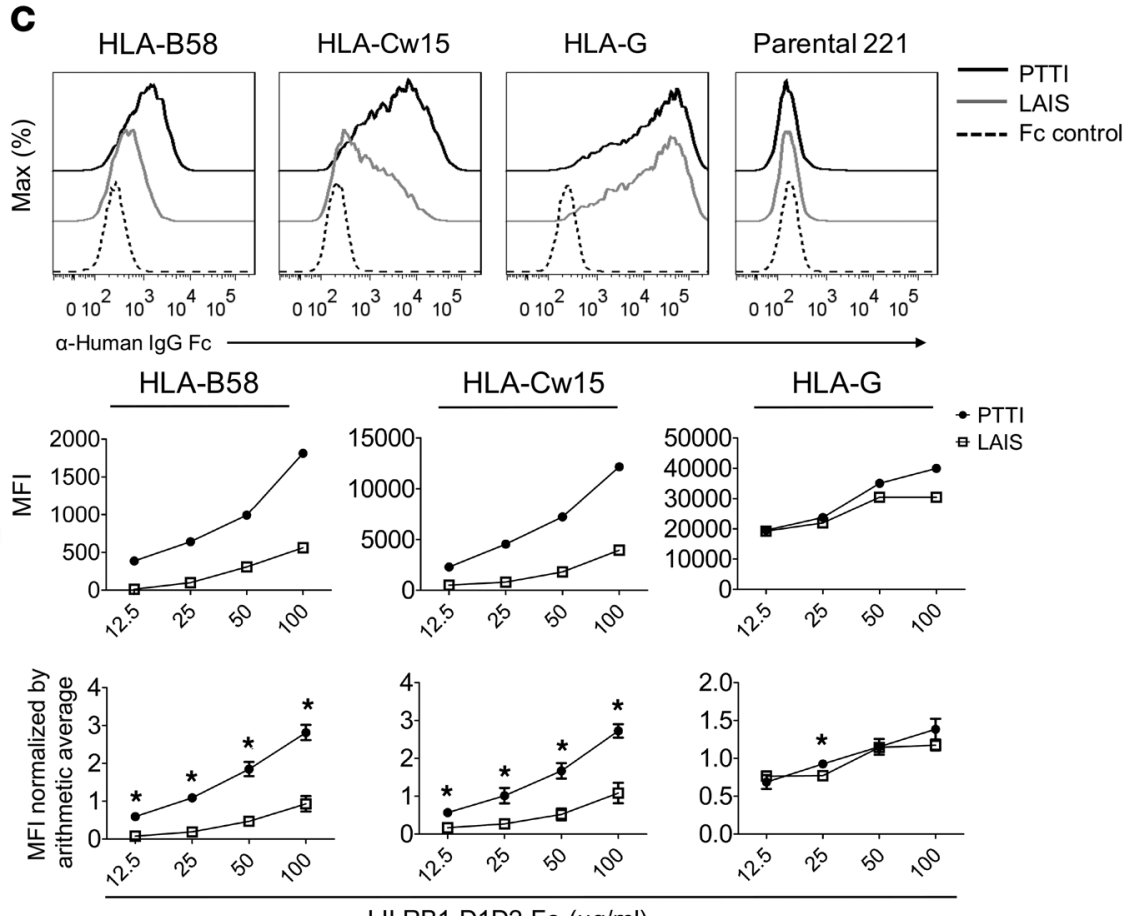

D

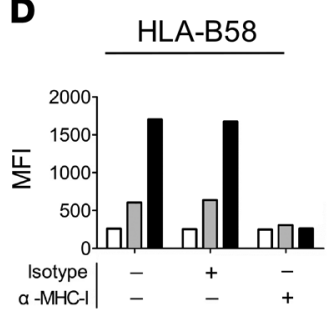

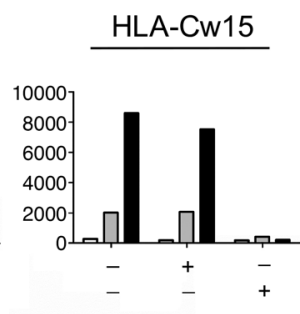

E

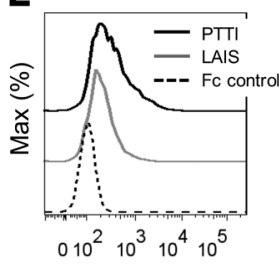
a-Human Ig G FC $\longrightarrow$

G

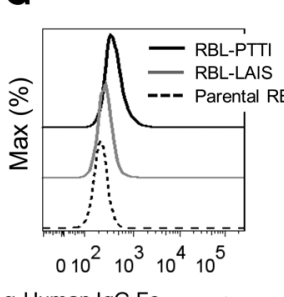

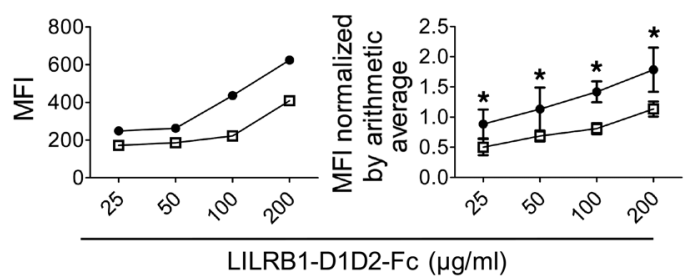

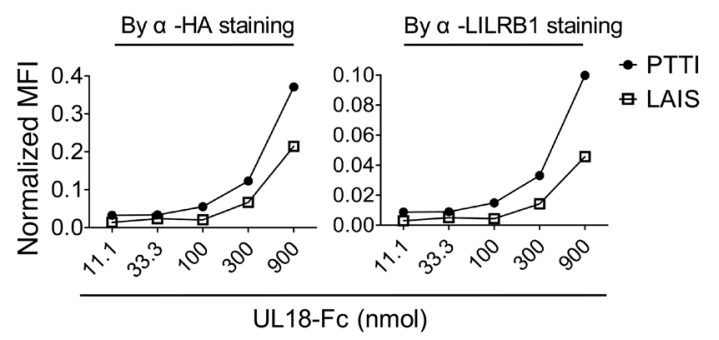

$\mathbf{F}$

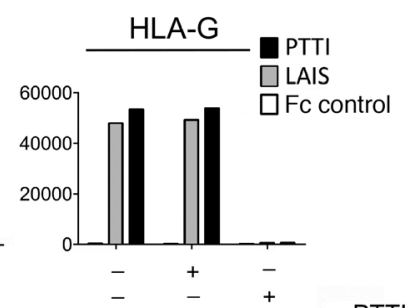

- PTTI $\because$ LAIS
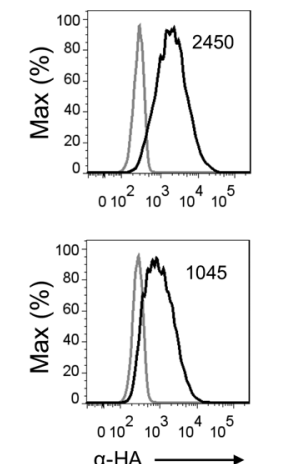
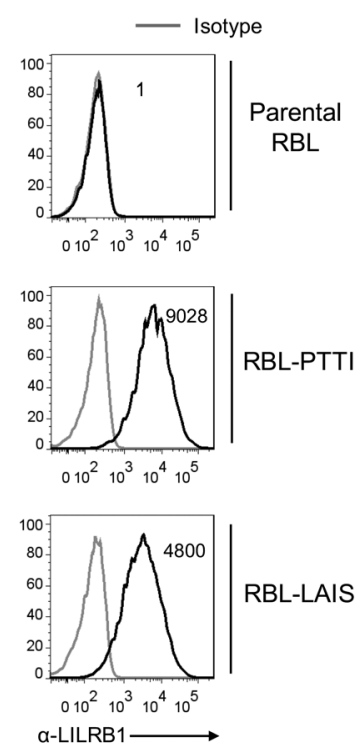
Figure 4. Binding of soluble LILRB1 variants to HLA-I molecules and HCMV-UL18. (A) Representative analysis of purified LILRB1 D1D2-FC fusion proteins by Coomassie blue staining (left) and $\alpha$-human IgC Fc Western blot (right). (B) Reactivity with $\alpha$-LILRB1 (HPF1) was determined by ELISA over the indicated range of concentration of the LILRB1-Fc protein. Results shown are the average of 3 independent tests for the same batch of protein; error bars represent SD. (C) The top histograms illustrate binding of purified LILRB1-Fc to 221 cells with HLA-B58, HLA-Cw15, and HLA-C by flow cytometry at $50 \mu \mathrm{g} / \mathrm{ml}$. The middle panels show 1 representative titration plotted as the MFI. The bottom series of plots show the normalized binding results aggregated from 3 independent tests. ${ }^{*} P<0.05$ using 1-way ANOVA. (D) Cells expressing HLA-I were incubated with $10 \mu \mathrm{g} / \mathrm{ml} \alpha$-MHCI (W6/32) or the isotype antibody before addition of $50 \mu \mathrm{g} / \mathrm{ml}$ LILRB1 variants or Fc control. The binding was measured by flow cytometry. The plots shown are a representative result of 3 independent tests. (E) Binding of LILRB1-D1D2-Fc variants to UL18 expressed on 721.221 cells. The flow histogram on the left shows the binding of UL18 with 100 $\mu \mathrm{g} / \mathrm{ml} \mathrm{Fc}$ fusion proteins. The middle plot shows a representative experiment across 4 concentrations. The far-right graph shows the aggregate data for 3 experiments normalized as described in Methods ( ${ }^{*} P<0.05$ using 1-way ANOVA). (F) Expression of the LILRB1-PTTI and -LAIS variants on transduced RBL cells was measured by $\alpha$-HA or $\alpha$-LILRB1 (HPF1) staining (representative of 3 independent tests). (G) UL18-Fc binding to LILRB1 variants expressed on RBL cells shown in $\mathbf{F}$ with a representative histogram shown on the left at $300 \mathrm{nmol}$. The binding data are normalized by the MFI for $\alpha$-HA (middle) or $\alpha$-LILRB1 (HPF1) (far right). The results are representative of 3 independent tests.

we mutated the asparagine residue at position 117 to glutamine (N117Q). The N117Q-PTTI-Fc receptor comigrated with LAIS-Fc, and the N117Q mutation in LAIS-Fc did not alter its mobility in a gel (Figure 5C). The N117Q mutation in the 2 mutants also did not change the reactivity with HPF1 as detected by ELISA (Figure 5D). As predicted, the binding of N117Q-PTTI to HLA-Cw15 was diminished to a level similar to LAIS (Figure 5E). N117Q-LAIS-Fc binding with Cw15 was unchanged compared with LAIS-Fc and was close to the limit of detection (Figure 5E). Somewhat unexpectedly, mutation of residue 117 to a glutamine in PTTI and LAIS reduced binding to HLA-G; however, the 2 mutants still bound similarly to HLA-G (Figure 5E). These observations suggest that in addition to being a site for glycosylation in PTTI, residue N117 in LAIS influences the interaction with HLA-G without being glycosylated. Surprisingly, N117Q substitution enhanced the binding of both LILRB1 variants to UL18, with similar binding curves (Figure 5E). These results point to the importance of residue 117 and its modification being important in the interaction of LILRB1 with various endogenous and viral ligands even if there is not an obvious explanation for the enhanced binding of the N117Q mutant.

All 4 residues contribute greatly to the interaction between UL18 and MHCI. To directly test whether residue T119 influences binding and is involved in the glycosylation of PTTI, we used sitedirected mutagenesis to generate the variants PTII and LTTI to test the role of residue P45 in this interaction. PTII comigrates with LAIS, confirming the effect of T119 on migration and glycosylation (Figure 6A). PTII does not alter reactivity with HPF1 (Figure 6B). Consistent with our prediction that T119 influences the interaction of Cw15 and UL18, binding of PTII-Fc to Cw15 and UL18 was reduced compared with PTTI (Figure 6C). Substitution of the proline residue at position 45 to leucine also significantly reduced the interaction with UL18 and Cw15, almost as much as the T119I mutation. These 2 mutations also reduced binding to HLA-G.

To address the role of T90 and I132, we generated the corresponding versions of the D1D2 domains with the combinations PATI and PTTS as Fc fusion proteins. As expected, PATI-Fc and PTTS-Fc migrated more slowly than LAIS-Fc, similar to PTTI-Fc (Figure 6D), and there was no difference in reactivity with HPF1 (Figure 6E). PATI bound to all ligands with similar efficiency as LAIS, with reduced binding to both HLA-Cw15 and UL18 (Figure 6 F). These results suggest that only NK cells with the PTTI variant would be well inhibited by UL18, and predict that PATI behaves similarly to LAIS, although it is associated with polymorphisms in the promoter region leading to low frequency of expression on NK cells. The presence of a serine at position 132 in the context of a receptor with PTT in the first 3 variable positions reduces the binding with UL18 relative to PTTI and, remarkably, has a dramatic enhancing effect on HLA-G binding (Figure 6F).

Predicted structural consequences of LILRB1 polymorphisms. We have demonstrated that substitution of each of the 4 residues significantly influences binding to both classical MHCI molecules and to the CMV-associated MHCI mimic UL18. In order to evaluate the structural consequences of the LILRB1 polymorphism on its interactions with each $\mathrm{MHC} /$ peptide, we created a 3D model for each MHC/peptide in complex with each LILRB1 variant (see Methods for details). Superposition of the 3 structurally determined LILRB1 variants demonstrates angle differences between the D1D2 domains, which could have a substantial impact on the capacity of each LILRB1 variant to interact with each studied MHC/peptide complex (Supplemental Figure 4B). Comparison of the 3 complexes (Figure 7, A-C) indicates that although the overall interaction is similar, several of the polymorphisms result in the large observed differences in affinity.

The main difference between HLA-A2 and HLA-G in their respective interaction with LILRB1-PATI is the sequence and conformation of a loop localized between the $\beta$ strands of the $\alpha 3$ domain. Indeed, the stretch of HLA-A2 residues (S195D196-H197) and the corresponding stretch of residues in HLA-G (F195-D196-Y197) are different in conformation and properties (Figure 7, D-F). These polymorphisms result in substantial changes in the side chains of 2 residues (S195F and H197Y), both in size and properties. Furthermore, the conformation of the loop is very different in HLA-G compared with classical HLA molecules, substantially altering the binding interface. These structural comparisons, although largely based on molecular modeling, provide a potential explanation for the differential binding of LILRB1 to UL18, HLACw15, and HLA-G.

Consistent with the binding data, the LILRB1 residue P45 plays an important role in interactions with UL18 (Figure 7D) in the model. Although P45 is localized at $4.2 \AA$ from the UL18 residue N199, it affects the conformation of the side chain of N199, facilitating the formation of a hydrogen bond between N199 and residue T43 in LILRB1. In addition, the small and rigid residue P45 allows UL18 residues N201 and D202 to come closer to LILRB1 and form a hydrogen bond with the backbone of L37 in LILRB1 and a salt bridge between the LILRB1 residue R84 and the UL18 residue D202 (Figure 7D). Comparison of LILRB1-LAIS (Protein Data Bank [PDB] code 1vdg) with any other LILRB1 structure indi- 
A

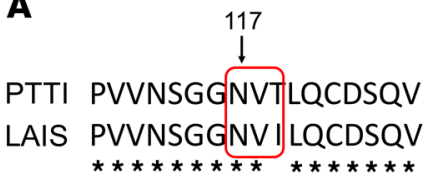

B

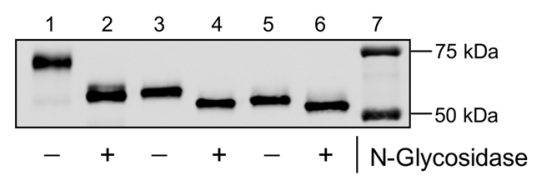

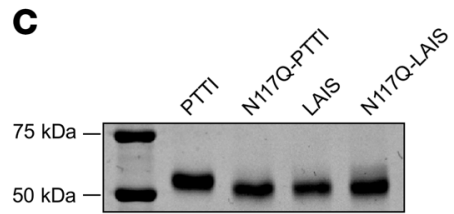

D

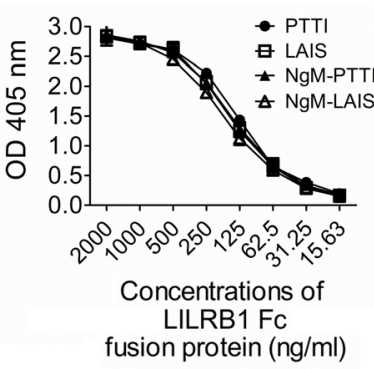

E

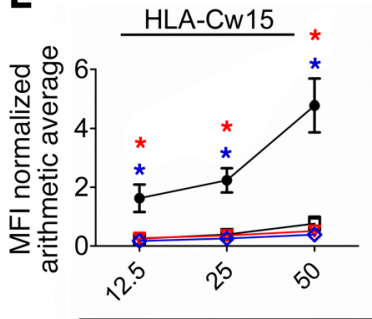

HLA-G

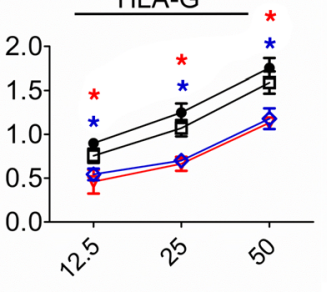

LILRB1-Fc and mutants (ug/ml)

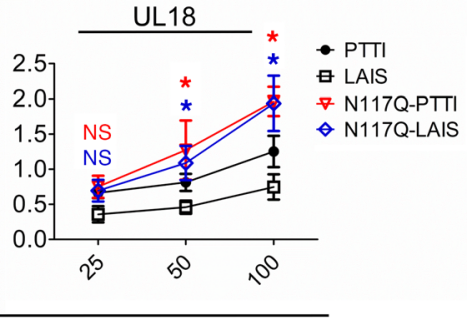

Figure 5. Mutation of the putative glycosylation site alters binding. (A) The sequence surrounding the putative N-linked glycosylation site NVT at position 117 is shown for both variants. The region boxed in red illustrates the target sequence NVT, present only in the variants with T at position 119. (B) LILRB1-PTTI and -LAIS variants treated with N-glycosidase analyzed by SDS-PAGE and Western blot. Lanes 1-2 are KIR3DL1-Fc, 3-4 are LILRB1-PTTI-Fc, 5-6 are LILRB1-LAIS-Fc, and 7 is the molecular weight marker. (C) Representative SDS-PAGE and Coomassie blue staining of the LILRB1-Fc N117Q-PTTI and N117Q-LAIS mutants. Lanes from left to right indicate the protein ladder, LILRB1-PTTI-Fc, N117Q-PTTI-Fc, LILRB1-LAIS-Fc, and N117Q-LAIS-Fc. (D) Reactivity with $\alpha$-LILRB1 (HPF1) for the mutated LILRB1 by ELISA. Results shown are the average of 3 independent tests for the same batch of protein; error bars represent SD. (E) Fc fusion protein binding to cells expressing the ligands at the top was measured by flow cytometry as before. Significance testing was performed between the binding of each artificial mutants and PTTI. ${ }^{*} P<0.05, \mathrm{~ns}=P \geq 0.05$ using 1-way ANOVA. The plots are the normalized binding results aggregated from at least 3 independent tests ( 4 and 5 independent tests for HLA- $G$ and UL18, respectively).

cated no significant structural changes in D1 or D2. Substitution of P45 to a leucine larger than P45 repels the UL18 residue N199 and distorts the interface established between UL18 and the LILRB1PATI variant.

The LILRB1 residue A70 is localized in the hinge region that connects the 2 immunoglobulin domains D1 and D2 in LILRB1 (Supplemental Figure 7A). Comparison of the crystal structures of the 2 LILRB1 alleles PATI and PTTI (Supplemental Figure 4B) reveals clear differences in the angle between D1 and D2. The flexibility between D1 and D2 is very important, since it is known that LILRB1 changes the interdomain angle by $15^{\circ}$ upon complex formation (17). In addition, the crystal structures show clear differences in the conformation of specific loops within the interdomain region, which are part of site 1 in interactions with UL18 and HLA-A2. The A70T polymorphism could directly impact the hinge region, since the hydroxyl group of T70 in PTTI forms a hydrogen bond with the carboxyl group of residue E184 in the D2 domain (Supplemental Figure 7A, left). This interaction not only can affect the angle formed between D1 and D2 and/or the flexibility of those domains, but it also alters the conformation of E184 (Supplemental Figure 7A, right). Residue E184 forms a strong salt bridge in the crystal structures of both the LILRB1/HLA-0201 and LILRB1/UL18 complexes $(25,26)$. It should also be noted that the substitution of $\mathrm{E} 184 \mathrm{~V}$ is considered to be one of the main causes underlying the distinct binding mode between LILRB1 and LILRB2 (27). Thus, the substitution of residue A70 to a threonine will indirectly alter the interaction between the LILRB1 variant PTTI and MHC molecules.

Finally, the functional effect of the isoleucine to serine mutation at position 132 can be explained by comparing the crystal structures of the PATI- and LAIS-LILRB1 alleles (Supplemental Figure 7B). The PATI- and LAIS-LILRB1 molecules have different conformations in several loops within the D2 domain. Residue N146 forms a hydrogen bond with Y177 and S132 in PATI and LAIS, respectively, resulting in marked conformational differences in the loop. In addition, the I132S substitution creates a cavity within the hydrophobic core of the D2 domain that allows residues W185 and Y177 to move closer to the C134-C144 disulfide bridge in order to fill this cavity in LAIS. This movement pulls residue E184 further away from the $\beta_{2}$ m residue K91, reducing the interaction of LAIS-LILRB1 with HLA/UL18.

\section{Discussion}

We initially speculated that low LILRB1 expression on NK cells would allow for enhanced control of HCMV infection due to reduced inhibition by the viral MHCI homolog UL18. In contrast to our predictions, we found that polymorphisms that predict lower frequency of expression on NK cells are directly associated with poor control of HCMV in transplant patients. The association of the alleles with lower expression can make sense because we have also shown that the LILRB1 protein variant encoded by one of the alleles interacts more strongly with UL18. We showed that individual substitution of each one of the 4 polymorphic amino acids in the ligand binding domains significantly alters the physical interaction of the LILRB1 receptor with UL18. Surprisingly, the polymorphisms also change the binding with classical MHCI proteins. The natural LILRB1 variants tested maintain strong interaction with HLA-G, whereas individual amino acid modification either reduces or enhances interaction with HLA-G. Together, these data indicate that LILRB1 interaction with HLA-G is a dominant selec- 
A

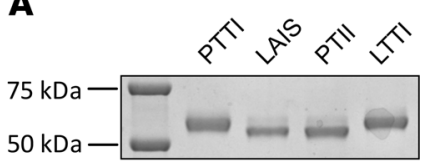

B

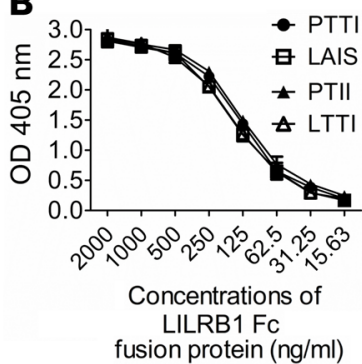

C

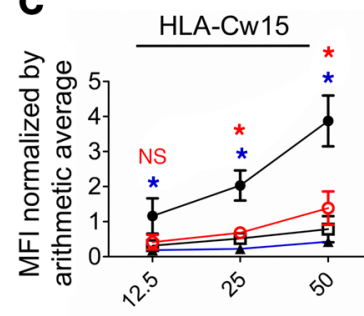

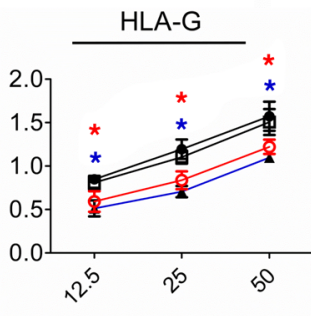
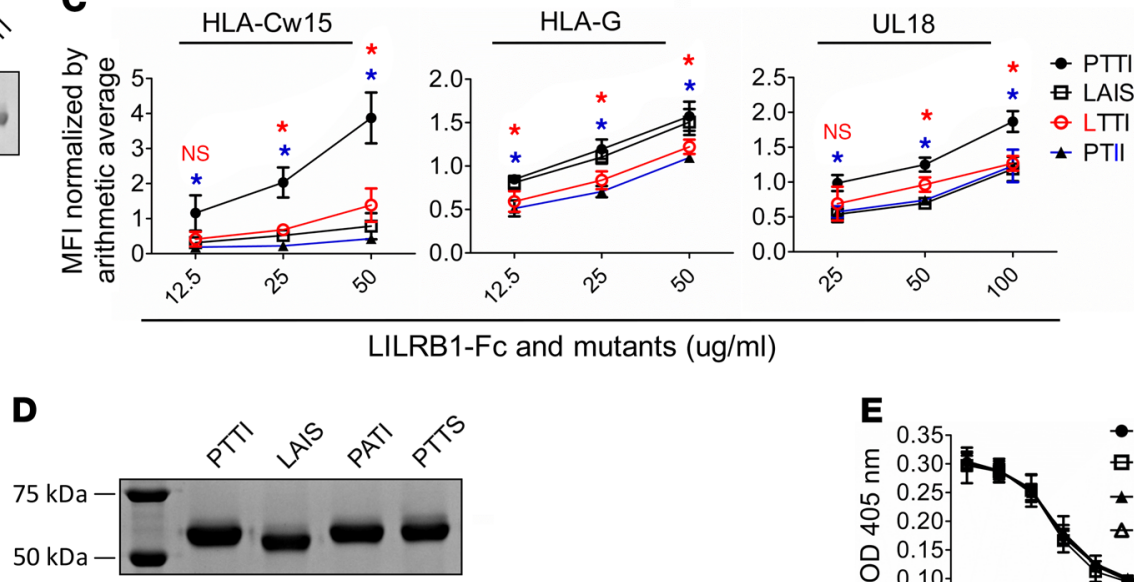

$\mathbf{F}$
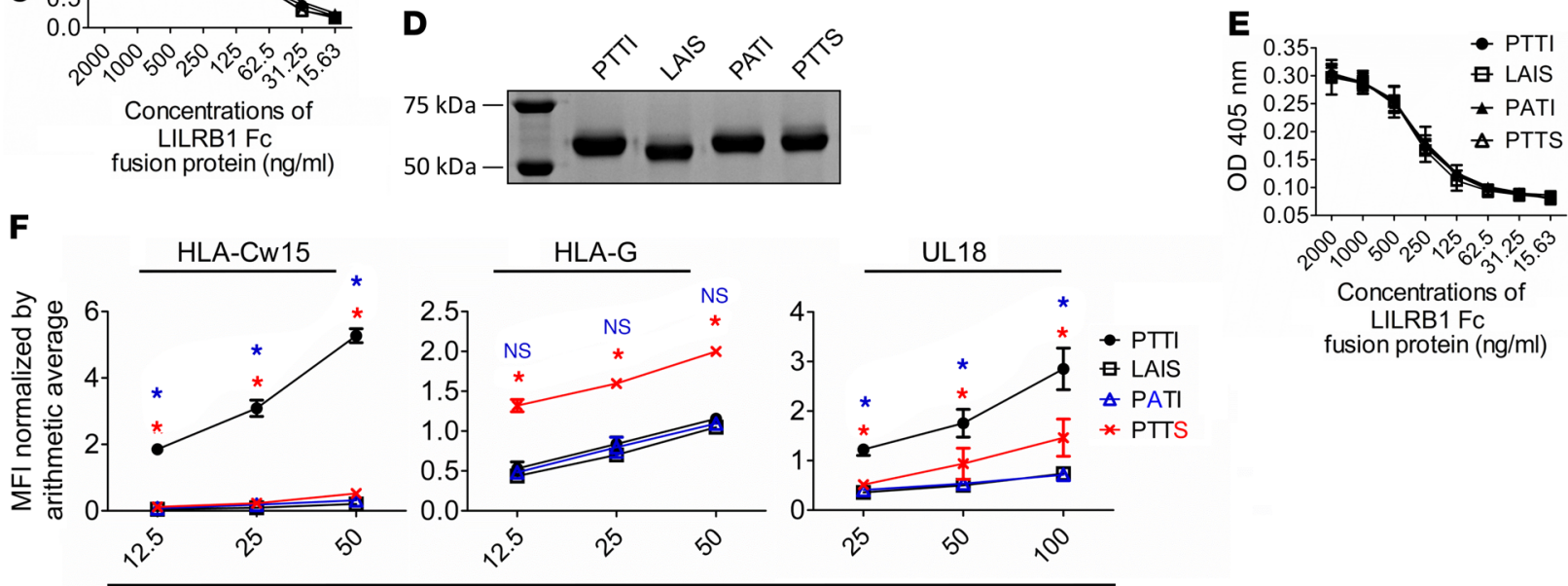

LILRB1-Fc and mutants (ug/ml)

Figure 6. Contributions of each residue to binding. (A and D) Migration of LILRB1-D1D2-Fc fusion protein mutants as detected by Coomassie blue staining. (B and E) Reactivity of the LILRB1-D1D2-Fc fusion proteins with $\alpha$-LILRB1 (HPF1) measured by ELISA. Results shown are the average of 3 independent tests for the same batch of protein; error bars represent SD. (C and F) Normalized binding aggregated from 3 independent tests. Significance testing was performed between the binding of each artificial mutant and PTTI. ${ }^{*} P<0.05$ using 1-way ANOVA.

tive force maintaining LILRB1 haplotypes, whereas alleles with higher affinity for UL18 have adapted through selective regulation of LILRB1 expression on NK cells in order to limit evasion of NK responses by HCMV. The structural analysis provides a possible explanation for how residues that are outside the binding interface influence the interaction between LILRB1 and UL18. It remains to be determined which polymorphism can best predict outcomes with HCMV and whether differences in interaction with classical MHCI influence the function of LILRB1 in other cell types and with other pathogens.

The rather dramatic effect caused by introducing an isoleucine at position 119 to generate PTII suggests that glycosylation influences binding, and likely explains why an earlier study did not detect any differences in affinity using proteins generated in bacteria (17). The glycan could interact directly with UL18, but it is more likely to contribute by stabilizing a particular conformation that favors binding, perhaps influencing the angle of the hinge. What remains difficult to explain is why introduction of glutamine into position 117 disrupted binding to Cw15 and HLA-G, while simultaneously enhancing binding to UL18 for both variants. It is possible that there is a steric hindrance with UL18 due to its glycosylation, which is not found on MHCI. Another possibility, although not mutually exclusive, is that the residues are part of a second functional interface of the receptor because all of the polymorphic residues are located on the same face. The near-total coverage by gly- cosylation of the UL18 surface outside the area of known LILRB1 interaction likely precludes additional points for contact. An additional possibility is that the residues influence the dimerization of the receptor in a manner similar to what has been suggested for the related KIR inhibitory receptors, where formation of higher-order complexes may be involved in signal transduction (28). However, it is difficult to imagine how formation of higher-order complexes would have similar effects for MHCI and UL18 but not HLA-G.

The similar binding that we observed of HLA-G with natural variants compared with the artificial combinations we generated suggests that maintaining interaction with HLA-G within a particular threshold provides selective pressure on diversification of LILRB1. HLA-G is best known for its expression in the placenta, where it is presumed to modulate the environment to prevent immune-mediated fetal rejection (29). Introduction of a serine at position132into the PTTIvariantsignificantlyaugmented binding to HLA-G, but this combination does not occur at any appreciable frequency as a natural variant (24). The LAII haplotype is carried by $2.7 \%$ of the European population; it is a variant likely to have lower affinity for HLA-G and that could influence reproduction. Perhaps LILRB1 polymorphism is relevant in cancer, as HLA-G expression on cancer cells is associated with poor outcomes $(30,31)$.

The overall effect of LILRB1 genotype on control of HCMV is relatively mild. The SNP with the highest significance differs between the 2 cohorts, although they both map the same extend- 
A

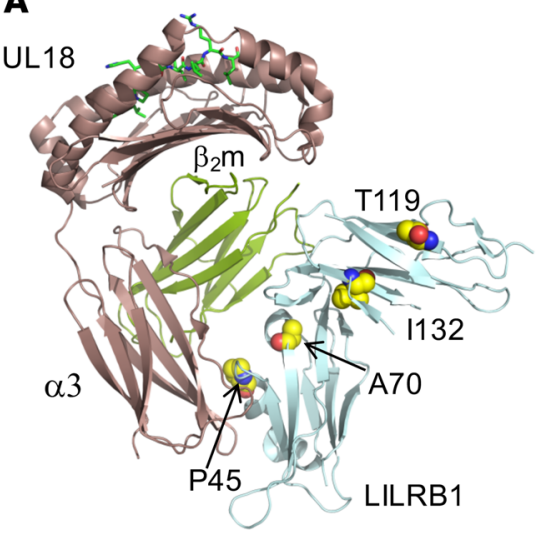

B

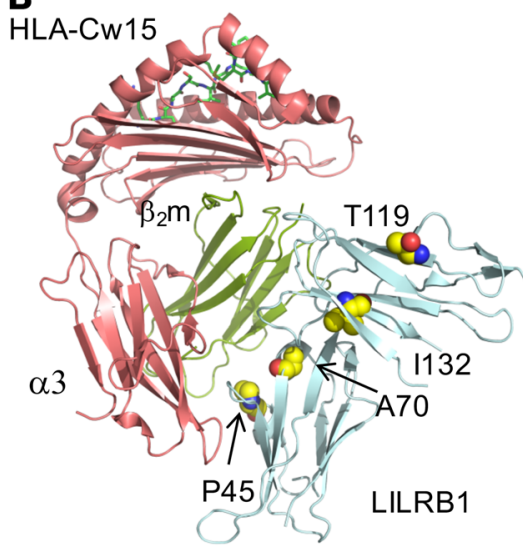

C

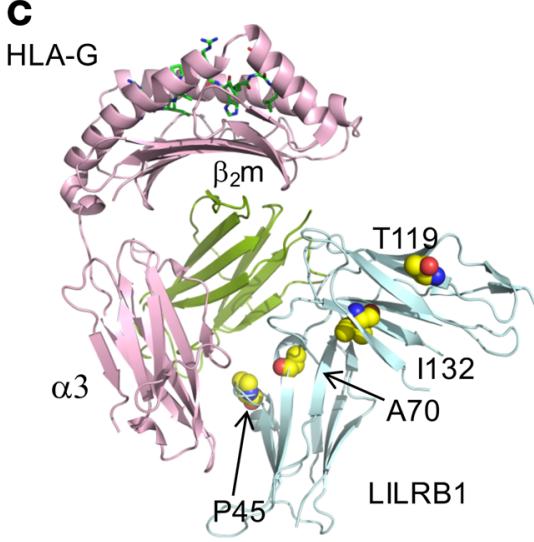

D

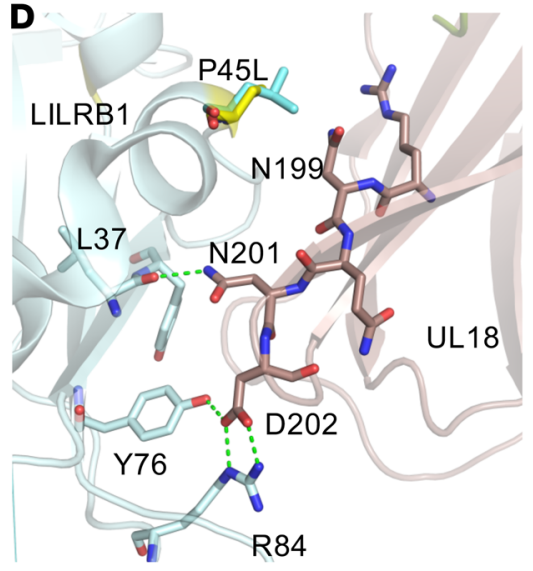

$\mathbf{E}$
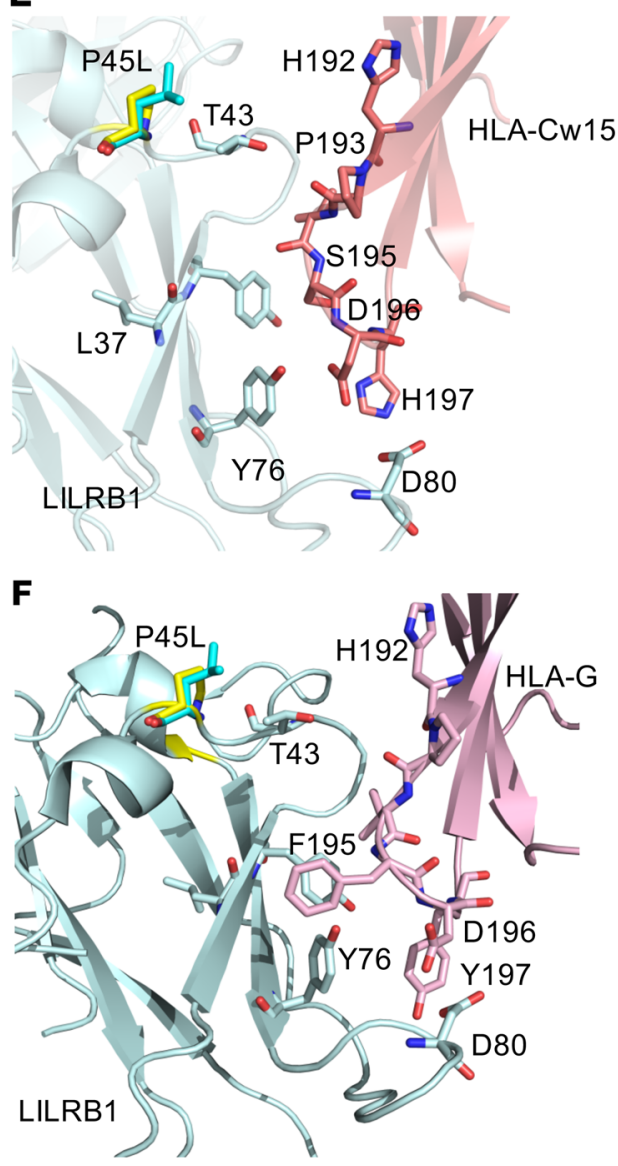

Figure 7. P45L has larger effects on the UL18/LILRB1 interaction compared with MHCI/LILRB1. Comparison of the 3D structures of 3 complexes, including the crystal structure of UL18/LILRB1 (A) and the molecular models of HLA-CW15/LILRB1 (B) and HLA-G/LILRB1, (C) illustrates the similarity of their binding modes as well as important differences in the details of these interactions. Proteins are displayed as cartoons with heavy chains of UL 18 and HLA molecules in different pink colors, the $\beta_{2} m$ subunit in green, and LILRB1 in light cyan. The 4 residues that differ between the LILRB1 alleles are shown as yellow spheres. (D) Conformation of P45L in LILRB1 interacting with UL18 compared with the complexes formed with HLA-Cw15 (E) or HLA-C (F). LILRB1 is in light cyan, and the chains of UL18 or HLA are pink. Residues important for the interactions are displayed as sticks. ed haplotype that correlates with less frequent LILRB1 expression on NK cells. Further dissecting which SNPs control expression in NK cells and which influence binding should refine any further examinations of the genetic association with CMV replication. Our results are in agreement with higher rates of HCMV disease in a subgroup of $\mathrm{HIV}^{+}$patients with a threonine at position 119 (18). Future studies that assess SNPs within the interval between $r s 1004443$ and $r s 1061680$ may locate the most predictive SNPs. For example, a stronger association may be revealed with removal of the variants encoding alanine at $r$ s124060501, as this subset has weak binding to UL18 and the frequency of PATI haplotypes is $12 \%$.
It is not entirely clear why the correlation between LILRB1 genotype and susceptibility to HCMV infection is pronounced in STCS kidney transplant patients or why a similar skewing for viremia was observed in the $\mathrm{D}+\mathrm{R}-$ group from Canada. The results of this study need to be viewed with the standard qualification for analysis of subgroups as well as the reduction of the sample size, but the results are also strengthened by the similarity of the outcomes between the 2 cohorts in terms of the extended haplotype that associates with more HCMV (disease or viremia). In both cohorts, the kidney is the predominant organ type, with the liver being the second most frequent organ type. The organ distribution has obvious implications, since liver recipients are typically 
at a lower risk for HCMV infection and disease, likely decreasing the strength of the association between HCMV replication and the genotypes when the overall cohorts (i.e., all organ types) are taken into consideration. In the Swiss cohort data set genotyped for rs10423364, the rate of HCMV infection in kidney recipients was $33.79 \%$ vs. $28.32 \%$ in recipients of other organ types. The Canadian patients were all HCMV D+/R-, with a much higher incidence of HCMV disease than what is seen in the Swiss cohort $(25 \%$ vs. $<6 \%$ ), in which approximately $76 \%$ of the recipients were HCMV seropositive. The difference may explain why in the latter group we see a genotype effect only for viremia. In a previous study using patients from the STCS, the association with the KIR genotype was seen predominantly in patients receiving the highest level of immune suppression-specifically, heart and lung recipients as well as kidney recipients given antithymocyte globulin (ATG) (32). However, we found KIR and LILRB1 to be independent variables in STCS samples despite their close proximity to one another within the leukocyte receptor complex.

Two previous studies might provide an alternative explanation for our results, as they suggest a potential protective influence of LAIS and the related variants. A very recent study showed that certain UL18 variants that differ from AD169 not only fail to inhibit NK cells but in fact stimulate LILRB1 ${ }^{+}$NK cells (16). The study did not take into account the LILRB1 variants, but it did show that the stimulation required LILRB1. Another, earlier study demonstrated that LILRB1 ${ }^{+} \mathrm{T}$ cells are stimulated through UL18 even though LILRB1 inhibits T cells in other situations, suggesting the signal transmitted by LILRB1 may be context dependent (33-38). Since many HCMV-specific effector/memory T cells are $\mathrm{LILRB1}^{+}$, the role of LILRB1 variation in $\mathrm{T}$ cell responses should be considered as well. The contribution of LILRB1 interaction with MHCI may also be a factor. In our earlier study, we found that few if any LILRB1 ${ }^{+} \mathrm{NK}$ cells respond to classical MHCI; however, it is unlikely a PTTI homozygous donor was tested (39).

The significance of the association of LILRB1 SNPs with HCMV disease is that it may be useful as part of a collection of biomarkers used to determine the relative risk for patients after receiving a transplant and to guide prophylactic use of antivirals in these patients. Many studies have investigated genetic influences on the immune response to HCMV; the genes implicated in modulating the control of HCMV after transplantation include $I L-28 B, C C L 8$, several microRNA, CLTA4, TLR9, DC-SIGN, and, most relevant to NK cells, NKG2C and KIRs (40-56). Further studies with additional cohorts could be useful to pinpoint the SNPs with the best correlation and to explore the relationship to the sequence of UL18 within each patient. Another interesting implication of the present work relates to prenatal and perinatal infection with HCMV. HCMV is the leading cause of birth defects resulting from infection, and estimates of permanent damage are as high as $0.1 \%$ of births. Deafness is the most frequent problem, and it is more prevalent in a primary infection (57). LILRB1 is expressed by myeloid cells within the decidua as well as NK cells (58), and LILRB1 variation might be a factor in controlling transmission of the virus to the fetus or infant.

Studies of mouse CMV (MCMV) contributed much to the understanding of adaptive NK cell responses and evolution of NK cell receptor systems in response to the virus (59-61). A substantial body of work has established the effect of HCMV infection on the NK cell repertoire in humans (reviewed in ref. 62). Our results demonstrate that a small change in affinity between LILRB1 and UL18 can greatly influence control of HCMV after transplantation. Our findings have opened many new questions about the potential effect of LILRB1 variation in other HCMV-mediated pathologies, and they emphasize the importance of maintaining an appropriate level of LILRB1 interaction with HLA-G. The apparent imprint of UL18 on the evolution of LILRB1 underscores the influence of highly adapted pathogens on the human genome and implies similar relationships with other pathogens that could shape the NK cell receptor repertoire.

\section{Methods}

\section{Genotyping of transplant patients}

The Canadian cohort SNP study consisted of a post hoc analysis of all the local patients included in a multicenter cohort study, and no Canadian patients were excluded from the post hoc analysis. Access to patient specimens from the remaining sites was considered to be impractical and was not pursued. All relevant information regarding sample size calculation, recruitment, enrollment, and inclusion/ exclusion criteria, as well as a flowchart summarizing the recruitment process for the entirety of the multicenter cohort study, is available in the original article (22). Briefly, patients were enrolled at the time of transplantation and received HCMV antiviral prophylaxis. Viremia was measured, as HCMV DNAemia and HCMV disease (63) were tracked for the first year after transplantation. Samples were genotyped using Taqman, and the associations were determined in isolation or in combination (i.e., heterozygous individuals with homozygous carriers of either the major or minor alleles). Associations between SNP genotypes and outcomes were investigated for the entire cohort and also separately for each participating center.

The association of LILRB1 SNP rs1061680 and its surrogate SNP rs10423364 ( $\left.r^{2} \sim 0.8\right)$ with HCMV infection or disease was determined in patients from the STCS (23). The STCS is a prospective, national cohort of SOT recipients involving 6 hospital centers in Switzerland (Lausanne, Geneva, Zurich, St. Gallen, Bern, Lugano, and Basel). Each SOT recipient within Switzerland who had signed, informed consent was recruited to the cohort. Each patient's clinical data were collected at baseline, 6 months after transplantation, and continue to be collected every 12 months after transplantation on standardized data forms by local physicians and data managers. Samples for genetic study (either whole blood or PBMCs) were collected from each patient at enrollment. CMV was monitored either in plasma or whole blood by using PCR arrays.

This study included all STCS SOT recipients recruited between May 2008 and November 2013, for whom all clinical data were available. In order to maintain consistency regarding allele frequencies, the analyses were limited to patients of European descent. Furthermore, patients who had incomplete or no genetic data and those whose DNA samples did not pass a quality check were excluded. Finally, we restricted our study to high-risk patients with a positive donor and/ or recipient CMV serostatus. Genotype data for both rs1061680 and rs10423364 were available for 1,018 STCS SOT recipients of European descent (776 with donor and/or recipient HCMV-positive serostatus; Table 1). Kidney recipients accounted for 651 of the study subjects (479 with donor and/or recipient HCMV-positive serostatus). An additional 100 patients had genotype data for $r s 1061680$ but not for $r s 10423364$. 


\section{Cell lines and antibodies}

YTS cells were obtained from Eric Long (NIH, Bethesda, Maryland, USA) as a gift and cultured with Iscove's modified Dulbecco's medium (IMDM) with $15 \%$ characterized FBS (ThermoFisher Scientific), 50 $\mu \mathrm{M} \beta$-mercaptoethanol, and $1 \mathrm{mM}$ L-glutamine. The human B lymphoma cell line 721.221 and 721.221 cells transfected with HLA-B58, HLA-Cw15, and HLA-G originally obtained through Eric Long were maintained in IMDM (Gibco, ThermoFisher Scientific) with 10\% FBS (Gibco, ThermoFisher Scientific) and supplemented with $0.5 \mathrm{mg} / \mathrm{ml}$ G418 sulfate (Life Technologies). Phoenix and Cos-7 cells were purchased from ATCC and cultured in DMEM (Gibco, ThermoFisher Scientific) with 10\% FBS. RBL cells were obtained from James Stafford (University of Alberta, Edmonton, Alberta, Canada) and maintained in MEM (ThermoFisher Scientific) containing 10\% FBS. Purified unconjugated mouse $\alpha$-human CD85j (clone: HPF1, IgG1к) (20) was provided by Miguel López-Botet (Pompeu Fabra University, Barcelona, Spain). Alexa Fluor 647-conjugated $\alpha$-HA (clone: 16B12) and IgG1 $\kappa$ isotype (clone: MOPC-21) were purchased from BioLegend. Unconjugated and APC-conjugated mouse $\alpha$-human CD85j (clone:

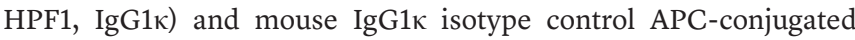

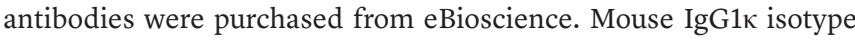
control (clone: MOPC-21) was purchased from Sigma-Aldrich. $\alpha-$ Human HLA-I (clone: W6/32) and $\alpha$-human CD8 (clone: 51.1) were purified from hybridoma supernatants (ATCC). Goat $\alpha$-human IgG Fc (catalog: 109-005-098) was from Jackson ImmunoResearch. Phycoerythrin (PE)-conjugated mouse $\alpha$-human IgG Fc (catalog: 9042-09) was from SouthernBiotech.

\section{Receptor constructs and transfections}

Full-length LILRB1 was amplified from the cDNA of a donor with known LAIS homozygous genotype. The coding sequence of the LILRB1 was amplified without the signal peptide using primers with restriction site additions (XmaI at the $5^{\prime}$ end and SalI at the $3^{\prime}$ end). The amplified genes were cloned into the TOPO pCRII vector using a TOPO-TA cloning kit (ThermoFisher Scientific). The LILRB1 gene was digested out of the pCR-II vector and cloned into the pDisplay expression vector (ThermoFisher Scientific), in frame with the HA tag. The PTTI variant of LILRB1 was produced using sequential site-directed mutagenesis of the LAIS variant in pCRII. Site-directed mutagenesis was done following the manufacturer's instructions for the QuikChange Lightning Mutagenesis kit (Agilent Technologies). Each mutation was confirmed by sequencing of the insert. The PTTI variant was digested out of the pCRII vector and cloned into the pDisplay vector.

$R B L$ transfection. RBL cells $\left(1.5 \times 10^{5}\right)$ were plated in a 6-well plate in $1 \mathrm{ml}$ of medium overnight. The medium was replaced with $250 \mu \mathrm{l}$ Opti-MEM (ThermoFisher Scientific) prior to addition of $1 \mu \mathrm{g}$ plasmid (HA-PTTI-LILRB1 or HA-LAIS-LILRB1 in pDisplay) and transfected using Xfect Transfection Reagent (Clontech) essentially according to the manufacturer's instructions. The cells were incubated for 4 hours at $37^{\circ} \mathrm{C} / 5 \% \mathrm{CO}_{2}$, and then $750 \mu \mathrm{l} \mathrm{RBL}$ media was added and the cells were cultured for another 48 hours. The medium was replaced with RBL medium containing $800 \mu \mathrm{g} / \mathrm{ml} \mathrm{G418.} \mathrm{G418-resistant} \mathrm{cells} \mathrm{were} \mathrm{sorted}$ by flow cytometry for matching levels of receptor using $\alpha$-HA antibody and continuously cultured in $800 \mu \mathrm{g} / \mathrm{ml} \mathrm{G418.} \mathrm{HA-tagged} \mathrm{LILRB1-}$ PTTI and -LAIS were subcloned into pMXs-puro vector (gift from Lewis Lanier, UCSF, San Francisco, California, USA) using ClaI and NotI restriction sites. YTS cells were transduced as previously described (64).
For the 221 cells with stable expression of HA-UL18-YFP, HCMV strain AD169-derived UL18 was provided by Lewis Lanier. The coding sequence was amplified with primers that replaced the stop codon with an in-frame sequence that allowed fusion to YFP using BamH1 and the pEYFP-N1 plasmid (Clontech) with linker sequence RDPPVAT. UL18-YFP was amplified by PCR with appropriate restriction sites and subcloned into pDisplay in frame with the N-terminal HA-tag generating a linker of GAQPARSPGIRGCRSS. The resulting construct was sequenced, and an apparent PCR error was corrected by site-directed mutagenesis. The construct was subcloned into pMXspuro (pMXs-HA-UL18-YFP). Phoenix cells $\left(2.5 \times 10^{6}\right)$ were plated in a $60-\mathrm{mm}$ dish for 16 hours and transfected with 8-15 $\mu$ g pMXs-HAUL18-YFP plasmid using calcium phosphate precipitation. After the incubation for 8 hours at $37^{\circ} \mathrm{C} / 5 \% \mathrm{CO}_{2}$, the medium was replaced with $3 \mathrm{ml} 721.221$ medium and cells were further incubated for 48 hours at $32^{\circ} \mathrm{C} / 5 \% \mathrm{CO}_{2}$. The supernatant was added to $5 \times 10^{5} 721.221$ cells with $8 \mu \mathrm{g} / \mathrm{ml}$ Polybrene (Sigma-Aldrich), plated in $1 \mathrm{ml}$ in a 24-well plate, spun at room temperature for 90 minutes at $365 \mathrm{RCF}$, and incubated for 3 hours at $37^{\circ} \mathrm{C} / 5 \% \mathrm{CO}_{2}$. The cells were pelleted again and cultured in fresh medium in a T25 flask for 48 hours and then selected with 1 $\mu \mathrm{g} / \mathrm{ml}$ puromycin (Bioshop Canada Inc.) replacing with fresh medium every 3-4 days. When cultures returned to confluence, UL18 surface expression was assessed by flow cytometry. Single-cell clones were obtained by the limiting dilution and surface expression detected with $\alpha$-HA antibody by flow cytometry.

LILRB1-Fc fusions and variants. The LAIS-LILRB1-D1D2-Fc has been previously described (65). For more efficient protein production we generated a new plasmid by moving the CD5 leader sequence and $\mathrm{Fc}$ region from CD5neg1 (gift from Eric Long) to the pEGFP (Clontech) backbone and removing the EGFP cassette (Fc-simple). The PTTI-LILRB1-D1D2 domains were moved into the $\mathrm{Fc}$-simple plasmid using PCR to generate an in-frame fragment with EcoR1 sites. The plasmids encoding the other LILRB1 mutants including PTII, LTTI, PATI, and PTTS were generated based on Fc-simple PTTI by site-directed mutagenesis.

\section{Cytotoxicity assay}

Effector cells were counted by TC20 Automated Cell Counter (BioRad Laboratories) and diluted to $10^{6}$ cells/ml with RPMI 1640 medium supplemented with 10\% FBS. Calcein-AM (Life Technologies) was dissolved in anhydrous DMSO (Life Technologies) at $100 \mu \mathrm{M}$ and stored for up to 1 week. Target cells were adjusted to $5 \times 10^{6}$ cells $/ \mathrm{ml}$ and labeled in the medium with $3.5 \mu \mathrm{M}$ calcein for 30 minutes at $37^{\circ} \mathrm{C} / 5 \%$ $\mathrm{CO}_{2}$. Calcein-labeled cells were washed twice with $10 \mathrm{ml} \mathrm{RPMI} \mathrm{medium}$ and counted and diluted to $5 \times 10^{4}$ cells/ml. For 10:1 of the E/T ratio reaction in each well of a 96-well plate, $50 \mu \mathrm{l} 10^{6}$ cells $/ \mathrm{ml}$ effector cells and $100 \mu \mathrm{L} 5 \times 10^{4}$ cells $/ \mathrm{ml}$ target cells were added in triplicate.

Where required, $50 \mu \mathrm{l}$ of $10 \mu \mathrm{g} / \mathrm{ml} \mathrm{HPF} 1$ or MOPC- 21 or medium were prealiquoted into a V-bottom 96-well plate (Corning). RPMI media $(100 \mu \mathrm{l})$ and/or RPMI containing $2 \%$ Triton X-100 were added to target cells for the spontaneous and maximum release, respectively. The plate was incubated at $37^{\circ} \mathrm{C} / 5 \% \mathrm{CO}_{2}$ for 4 hours and spun at 6 RCF for 2 minutes. Supernatant $(100 \mu \mathrm{l})$ was transferred to the black flat-bottom 96-well plate (Corning), and calcein release was measured in the EnSpire 2300 Multilabel Reader (PerkinElmer). Specific lysis was calculated based on the formula for each well: $100 \times($ release read-spontaneous release) / (maximum releasespontaneous release). 


\section{Purification of LILRB1-D1D2-Fc fusion protein}

Plasmids containing the variants and artificial mutants of LILRB1 D1D2 domains were amplified in E. coli and extracted by GenElute HP Plasmid Midiprep Kit (Sigma-Aldrich). Cos-7 cells seeded in T150 flasks were transfected with $20 \mu$ g DNA per flask. Cells were washed with DMEM 3 times at 12 hours after transfection and then maintained in serum-free DMEM with 1\% NEAA (ThermoFisher Scientific). Medium was collected and changed every 3 days (3 times) and filtered for affinity purification. The medium was loaded to a column filled with Protein $\mathrm{A} / \mathrm{G}$ beads (Millipore) at $4^{\circ} \mathrm{C}$, and the column was washed with PBS and then eluted by $0.05 \mathrm{M}$ glycine. Eluted protein was neutralized by $1 \mathrm{M}$ Tris buffer and dialyzed into PBS with Amicon centrifugal filters (Millipore). A protease inhibitor cocktail (Roche) was subsequently added to the purified protein. Concentration was determined using a Micro BCA kit (ThermoFisher Scientific). Purity and specificity were verified by Coomassie blue staining and Western blotting, respectively, using $\alpha$-human IgG Fc. Capturebased ELISA was used to check the conformation consistency of the purified LILRB1 variants and artificial mutants using HPF1, which targets the D1 and D2 domains of LILRB1 (64). Purified human IgG Fc fragment control has been previously described (64).

\section{LILRB1 binding assay}

The 221 cells were counted and their concentration adjusted to $2 \times$ $10^{7} / \mathrm{ml}$ with FACs buffer (2\% FBS and $1 \mathrm{mM}$ EDTA in PBS). Fc fusion protein $(10 \mu \mathrm{l})$ was added to $10 \mu \mathrm{l}$ cells in a 1.5-ml Microfuge (ThermoFisher Scientific) tube and incubated for 1 hour at $4^{\circ} \mathrm{C}$ with rotation, washed with $4 \mathrm{ml} \mathrm{FACs} \mathrm{buffer,} \mathrm{and} \mathrm{then} \mathrm{incubated} \mathrm{with} 100 \mu \mathrm{l} 5 \mu \mathrm{g} / \mathrm{ml}$ PE mouse $\alpha$-human IgG Fc for 30 minutes at $4^{\circ} \mathrm{C}$. Cells were washed again, fixed with $4 \%$ formaldehyde, and analyzed on an LSRFortessa analyzer (Becton Dickinson). Compensation was required for the YFP spill into the PE channel for binding to UL18-expressing cells. Where indicated, $100 \mu \mathrm{l} 10 \mu \mathrm{g} / \mathrm{ml} \mathrm{W6} / 32$ or isotype control was preincubated with the cells at $4^{\circ} \mathrm{C}$ for 10 minutes, and the cells were washed with FACs buffer prior to adding the $\mathrm{Fc}$ fusion protein.

The UL18-Fc fusion was generated as previously described (66). To account for the difference in the expression of LILRB1-PTTI and -LAIS variants on RBL cells, the binding was normalized by staining LILRB1 with $\alpha$-HA or HPF1.

\section{Deglycosylation}

Potential N-linked glycosylation sites were predicted with GlycoEP (IMTECH, http://www.imtech.res.in/raghava/glycoep/index.html). For enzymatic deglycosylation, $2 \mu \mathrm{g}$ purified proteins was adjusted to $43 \mu \mathrm{l}$ with $250 \mathrm{mM} \mathrm{NaHPO}_{4}$ buffer (pH 7.5) and then mixed with 2.5 $\mu \mathrm{l}$ denaturing buffer containing $2 \%$ SDS and $1 \mathrm{M} \beta$-mercaptoethanol, and incubated at $100^{\circ} \mathrm{C}$ for 5 minutes. The mixture was cooled to room temperature, and $2.5 \mu \mathrm{l} 10 \%$ Triton X-100 detergent was added with $2 \mu \mathrm{l}$ N-glycosidase F from Elizabethkingia meningosepticum (Millipore) and incubated for 3 hours at $37^{\circ} \mathrm{C}$. Samples were separated by $10 \%$ SDS-PAGE and stained by Coomassie blue.

\section{Three-dimensional modeling}

The crystal structures of the LILRB1/HLA-A2 and LILRB1/UL18 complexes have been previously determined using the PATI-LILRB1 variant $(25,26)$. We created 3D models of LILRB1/HLA-Cw15 and LILRB1/HLA-G based on the crystal structures mentioned above.
All modeling was performed manually in Coot (67). The 3D model of LILRB1/HLA-Cw15 was created using LILRB1/HLA-A2 as a template. The 3-domain of HLA-Cw15 differs from HLA-A2 in only 4 residues, and none of these polymorphisms are present in the HLA/ LILRB1 contact area. Thus, we hypothesized that the HLA-Cw15 binding mode should be similar to that of HLA-A2. We created the model of the HLA-G/LILRB1 complex based on the crystal structure of LILRB1/HLA-A2, replacing HLA-A2 with HLA-G (27).

In order to evaluate the structural consequences of the LILRB1 polymorphism, we created several 3D models. Crystal structures are available for 2 other allelic variants of LILRB1: LAIS (PDB code 1vdg) and PTTI (PDB code 1ugn) (17). Substitution of the PATI variant in the crystal structures of LILRB1/UL18 and LILRB1/HLA-A2 with LAIS or PTTIallowed us to evaluate theimpact of these polymorphic residues on the structure of the complexes. Following replacement of the subunits in the models, energy minimization was performed using the sphereregularization option in Coot. The model geometry as well as the conformation of the main chain (Ramachandran plot) were validated for all models in Coot. PyMol was used for preparation of all structural figures (PyMol Molecular Graphics System, version 1.5.0.4, Schrödinger).

\section{Statistics}

Two-tailed Pearson $\chi^{2}$ or Fisher's exact test was utilized to test for associations between categorical variables. Log-rank test was utilized to compare the Kaplan-Meier curves showing disease-free survival rates for HCMV DNAemia or disease. The associations of LILRB1 polymorphism and HCMV infection or disease were assessed by using univariate log-rank test and Cox regression models with censoring at 12 months after transplantation or until the date when the patient was lost to follow-up or died. In the multivariate Cox regression model, genetic associations were adjusted for relevant covariates as identified in a previous genetic association study of HCMV infection in the same cohort (40). Those variables included donor/recipient age, sex, and any appropriate covariates associated with the endpoint. Genetic associations were assessed using the recessive mode of inheritance (patients carrying 2 copies of the minor alleles are compared with the others). KIR genotypes were also used as covariates in a subset of 590 SOT recipients (including 263 kidney recipients) who had available genotypes. The LD, HardyWeinberg equilibrium (HWE) test, and minor allele frequency (MAF) were assessed by using the pwld and hwe software implemented in Stata 14 (StataCorp). The cutoff of $r^{2}>0.8$ was used to denote strong LD between studied polymorphisms. All SNPs passed the HWE test. For YTS killing assay, the means of the calculated specific lysis rates were compared by 2 -tailed paired samples $t$ test with significance of $P<0.05$. For the flow cytometry-based binding assay, each mean fluorescence intensity (MFI) value was corrected by subtraction of the Fc control MFI and then divided by the arithmetic average of the entire sample MFI in 1 independent test. The results of the binding assay were aggregated from at least 3 independent tests, and 1-way ANOVA was used to compare the binding of different purified proteins with a specific ligand. $P$ values less than 0.05 were considered significant. Statistical analysis was performed with SPSS Statistics 20 (IBM) and Prism v5.0 (GraphPad). Except as otherwise indicated, the experimental data are presented as mean $\pm S D$.

\section{Study approval}

Initial IRB approval for the Canadian cohort was obtained on January 30, 2009, under study ID Pro00002140. Additional IRB 
approval for use of specimens for the present study was sought and obtained on July 12, 2012. The participants provided informed written consent in all cases. The STCS protocol was approved by the independent ethics committees of each Swiss participating center: University Hospital of Lausanne (CHUV); University Hospitals of Geneva (HUG); University Hospital Zurich (USZ); Cantonal Hospital St. Gallen (KSSG); Inselspital, Bern University Hospital; Clinica Luganese, Lugano; University Hospital of Basel. All patients signed a written informed consent for participation in the STCS (including genetic analyses).

\section{Author contributions}

$\mathrm{DNB}, \mathrm{AH}$, and PYB conceived of and supervised portions of the study. KY, CLD, LL, AW, JV, AE, and TW participated in the study design. $\mathrm{KB}, \mathrm{AC}, \mathrm{CVD}, \mathrm{OM}, \mathrm{NM}$, and $\mathrm{KY}$ were involved in the STCS study design and sample collection. CLD, AW, and TW performed the experiments. AMA and JB generated material for the experiments. KY, CLD, LL, AW, JV, and TS performed the data analysis and interpretation. DNB, KY, LL, AW, PYB, and AA contributed to the writing of the manuscript. AH and AMA provided feedback on the manuscript.

\section{Acknowledgments}

This work was supported by funding from CIHR (MOP 123257), Li Ka Shing Institute of Virology (to DNB and AH), STCS (to DNB, CD, AW, and PYB), NSERC (to CD), and The China Scholarship Council (to KY). Part of this study has been conducted in the framework of the Swiss Transplant Cohort Study, supported by the Swiss National Science Foundation and the Swiss University Hospitals (G15) and transplant centers. We thank Lewis Lanier and Miguel Lopez-Botet for providing plasmids and antibodies, the contribution of the staff of the Flow Cytometry Core in the Faculty of Medicine and Dentistry at the University of Alberta, and Heather Eaton for technical support and editing of the manuscript. We thank Jim Smiley, Rob Ingham, Bart Hazes, and all the members of the Burshtyn group for helpful discussions. We graciously acknowledge support from Lorne Tyrrell for pursuing the project. We thank the patients for participating in the studies.

Address correspondence to: Deborah N. Burshtyn, Department of Medical Microbiology and Immunology, 6-020, Katz Building, University of Alberta, Edmonton, AB T6G 2S2, Canada. Phone: 780.492.0646; Email: burshtyn@ualberta.ca.
1. Orange JS. Unraveling human natural killer cell deficiency. J Clin Invest. 2012;122(3):798-801.

2. Lisnić B, Lisnić VJ, Jonjić S. NK cell interplay with cytomegaloviruses. Curr Opin Virol. 2015;15:9-18.

3. Muntasell A, Vilches C, Angulo A, López-Botet M. Adaptive reconfiguration of the human NKcell compartment in response to cytomegalovirus: a different perspective of the host-pathogen interaction. Eur J Immunol. 2013;43(5):1133-1141.

4. Tesi B, Schlums H, Cichocki F, Bryceson YT. Epigenetic regulation of adaptive NK cell diversification. Trends Immunol. 2016;37(7):451-461.

5. Jackson SE, Mason GM, Wills MR. Human cytomegalovirus immunity and immune evasion. Virus Res. 2011;157(2):151-160.

6. Cosman D, et al. A novel immunoglobulin superfamily receptor for cellular and viral MHC class I molecules. Immunity. 1997;7(2):273-282.

7. Davidson CL, Li NL, Burshtyn DN. LILRB1 polymorphism and surface phenotypes of natural killer cells. Hum Immunol. 2010;71(10):942-949.

8. Beck S, Barrell BG. Human cytomegalovirus encodes a glycoprotein homologous to MHC class-I antigens. Nature. 1988;331(6153):269-272.

9. Bjorkman PJ, Saper MA, Samraoui B, Bennett WS, Strominger JL, Wiley DC. Structure of the human class I histocompatibility antigen, HLA-A2. Nature. 1987;329(6139):506-512.

10. Bellón T, Kitzig F, Sayós J, López-Botet M. Mutational analysis of immunoreceptor tyrosinebased inhibition motifs of the Ig-like transcript 2 (CD85j) leukocyte receptor. J Immunol. 2002;168(7):3351-3359.

11. Chapman TL, Heikeman AP, Bjorkman PJ. The inhibitory receptor LIR-1 uses a common binding interaction to recognize class I MHC molecules and the viral homolog UL18. Immunity. 1999;11(5):603-613.

12. Romo N, et al. Association of atherosclerosis with expression of the LILRB1 receptor by human NK and T-cells supports the infectious burden hypothesis. Arterioscler Thromb Vasc Biol. 2011;31(10):2314-2321.

13. Wagner CS, Riise GC, Bergström T, Kärre K, Carbone E, Berg L. Increased expression of leukocyte Ig-like receptor-1 and activating role of UL18 in the response to cytomegalovirus infection. JImmunol. 2007;178(6):3536-3543.

14. Lopez-Vergès $S$, et al. Expansion of a unique $\mathrm{CD} 7^{+} \mathrm{NKG} 2 \mathrm{Chi}$ natural killer cell subset during acute human cytomegalovirus infection. Proc Natl Acad Sci U S A. 2011;108(36):14725-14732.

15. Cerboni C, et al. Spontaneous mutations in the human CMV HLA class I homologue UL18 affect its binding to the inhibitory receptor LIR-1/ILT2/ CD85j. Eur J Immunol. 2006;36(3):732-741.

16. Chen KC, Stanton RJ, Banat JJ, Wills MR. Leukocyte immunoglobulin-like receptor 1-expressing human Natural Killer cell subsets differentially recognize isolates of human cytomegalovirus through the viral major histocompatibility complex class I homolog UL18. J Virol. 2016;90(6):3123-3137.

17. Kuroki K, et al. Extensive polymorphisms of LILRB1 (ILT2, LIR1) and their association with HLA-DRB1 shared epitope negative rheumatoid arthritis. Hum Mol Genet. 2005;14(16):2469-2480.

18. Affandi JS, et al. Can immune-related genotypes illuminate the immunopathogenesis of cytomegalovirus disease in human immunodeficiency virus-infected patients? Hum Immunol. 2012;73(2):168-174.

19. Burshtyn DN, Morcos C. The expanding spectrum of ligands for leukocyte Ig-like receptors. J Immunol. 2016;196(3):947-955.

20. Colonna M, et al. A common inhibitory receptor for major histocompatibility complex class I molecules on human lymphoid and myelomonocytic cells. J Exp Med. 1997;186(11):1809-1818.

21. Li NL, Davidson CL, Humar A, Burshtyn DN. Modulation of the inhibitory receptor leukocyte Ig-like receptor 1 on human natural killer cells.
Front Immunol. 2011;2:46.

22. Manuel O, et al. Assessment of cytomegalovirusspecific cell-mediated immunity for the prediction of cytomegalovirus disease in high-risk solid-organ transplant recipients: a multicenter cohort study. Clin Infect Dis. 2013;56(6):817-824.

23. Koller MT, et al. Design and methodology of the Swiss Transplant Cohort Study (STCS): a comprehensive prospective nationwide long-term followup cohort. Eur J Epidemiol. 2013;28(4):347-355.

24. 1000 Genomes Project Consortium, et al. A global reference for human genetic variation. Nature. 2015;526(7571):68-74.

25. Willcox BE, Thomas LM, Bjorkman PJ. Crystal structure of HLA-A2 bound to LIR-1, a host and viral major histocompatibility complex receptor. Nat Immunol. 2003;4(9):913-919.

26. Yang Z, Bjorkman PJ. Structure of UL18, a peptide-binding viral MHC mimic, bound to a host inhibitory receptor. Proc Natl Acad Sci US A. 2008;105(29):10095-10100.

27. Clements CS, et al. Crystal structure of HLA-G: a nonclassical MHC class I molecule expressed at the fetal-maternal interface. Proc Natl Acad Sci US A. 2005;102(9):3360-3365.

28. Kumar S, et al. Zinc-induced polymerization of killer-cell Ig-like receptor into filaments promotes its inhibitory function at cytotoxic immunological synapses. Mol Cell. 2016;62(1):21-33.

29. Hunt JS, Petroff MG, McIntire RH, Ober C. HLA$\mathrm{G}$ and immune tolerance in pregnancy. FASEB J. 2005;19(7):681-693.

30. Rouas-Freiss N, Moreau P, Ferrone S, Carosella ED. HLA-G proteins in cancer: do they provide tumor cells with an escape mechanism? Cancer Res. 2005;65(22):10139-10144.

31. Carosella ED, Moreau P, Lemaoult J, RouasFreiss N. HLA-G: from biology to clinical benefits. Trends Immunol. 2008;29(3):125-132.

32. Gonzalez A, et al. KIR-associated protection from CMV replication requires pre-existing immunity: 
a prospective study in solid organ transplant recipients. Genes Immun. 2014;15(7):495-499.

33. Saverino $D$, et al. Specific recognition of the viral protein UL18 by CD85j/LIR-1/ILT2 on CD8 ${ }^{+} \mathrm{T}$ cells mediates the non-MHC-restricted lysis of human cytomegalovirus-infected cells. J Immunol. 2004;172(9):5629-5637.

34. Ince MN, et al. Increased expression of the natural killer cell inhibitory receptor CD85j/ILT2 on antigen-specific effector CD8 $\mathrm{T}$ cells and its impact on CD8 T-cell function. Immunology. 2004;112(4):531-542.

35. Saverino D, et al. The CD85/LIR-1/ILT2 inhibitory receptor is expressed by all human $\mathrm{T}$ lymphocytes and down-regulates their functions. J Immunol. 2000;165(7):3742-3755.

36. Saverino D, Merlo A, Bruno S, Pistoia V, Grossi CE, Ciccone E. Dual effect of CD85/leukocyte Ig-like receptor-1/Ig-like transcript 2 and CD152 (CTLA-4) on cytokine production by antigen-stimulated human T cells. J Immunol. 2002;168(1):207-215.

37. Costa P, et al. Differential disappearance of inhibitory natural killer cell receptors during HAART and possible impairment of HIV-1-specific CD8 cytotoxic T lymphocytes. AIDS. 2001;15(8):965-974.

38. Shiroishi M, et al. Human inhibitory receptors Ig-like transcript 2 (ILT2) and ILT4 compete with CD8 for MHC class I binding and bind preferentially to HLA-G. Proc Natl Acad Sci U S A. 2003;100(15):8856-8861.

39. Kirwan SE, Burshtyn DN. Killer cell Ig-like receptor-dependent signaling by Ig-like transcript 2 (ILT2/CD85j/LILRB1/LIR-1). J Immunol. 2005;175(8):5006-5015.

40. Manuel O, et al. Influence of IFNL3/4 polymorphisms on the incidence of cytomegalovirus infection after solid-organ transplantation. J Infect Dis. 2015;211(6):906-914.

41. Egli A, et al. Immunomodulatory function of interleukin 28B during primary infection with cytomegalovirus. J Infect Dis. 2014;210(5):717-727.

42. Corrales I, et al. IL28B genetic variation and cytomegalovirus-specific T-cell immunity in allogeneic stem cell transplant recipients. J Med Virol. 2017;89(4):685-695.

43. Cook M, et al. Donor KIR genotype has a major influence on the rate of cytomegalovirus reactivation following T-cell replete stem cell transplantation. Blood. 2006;107(3):1230-1232.
44. Hadaya K, et al. Natural killer cell receptor repertoire and their ligands, and the risk of CMV infection after kidney transplantation. Am J Transplant. 2008;8(12):2674-2683.

45. Jones DC, et al. Killer immunoglobulin-like receptor gene repertoire influences viral load of primary human cytomegalovirus infection in renal transplant patients. Genes Immun. 2014;15(8):562-568.

46. Li T, Dai W, Lu L. Ultraviolet-induced junD activation and apoptosis in myeloblastic leukemia ML-1 cells. J Biol Chem. 2002;277(36):32668-32676.

47. Loeffler J, et al. Polymorphisms in the genes encoding chemokine receptor 5 , interleukin-10, and monocyte chemoattractant protein 1 contribute to cytomegalovirus reactivation and disease after allogeneic stem cell transplantation. J Clin Microbiol. 2006;44(5):1847-1850.

48. Gumá M, Angulo A, Vilches C, Gómez-Lozano N, Malats N, López-Botet M. Imprint of human cytomegalovirus infection on the NK cell receptor repertoire. Blood. 2004;104(12):3664-3671.

49. Niknam A, et al. The association between viral infections and co-stimulatory gene polymorphisms in kidney transplant outcomes. Jundishapur J Microbiol. 2016;9(8):e31338.

50. Palumbo K, et al. The transcription factor JunD mediates transforming growth factor $\beta$-induced fibroblast activation and fibrosis in systemic sclerosis. Ann Rheum Dis. 2011;70(7):1320-1326.

51. Fernández-Ruiz M, et al. Association between individual and combined SNPs in genes related to innate immunity and incidence of CMV infection in seropositive kidney transplant recipients. Am J Transplant. 2015;15(5):1323-1335.

52. Misra MK, Pandey SK, Kapoor R, Sharma RK, Agrawal S. Cytotoxic T-lymphocyte antigen 4 gene polymorphism influences the incidence of symptomatic human cytomegalovirus infection after renal transplantation. Pharmacogenet Genomics. 2015;25(1):19-29.

53. Saadi MI, Yaghobi R, Karimi MH, Geramizadeh B, Ramzi M, Zakerinia M. Association of the costimulatory molecule gene polymorphisms and active cytomegalovirus infection in hematopoietic stem cell transplant patients. Mol Biol Rep. 2013;40(10):5833-5842.

54. Redondo-Pachón D, et al. Adaptive NKG2C+ NK cell response and the risk of cytomegalovirus infection in kidney transplant recipients. JImmu- nol. 2017;198(1):94-101.

55. Lisboa LF, et al. CCL8 and the immune control of cytomegalovirus in organ transplant recipients. Am J Transplant. 2015;15(7):1882-1892.

56. Berg L, et al. LIR-1 expression on lymphocytes, and cytomegalovirus disease in lung-transplant recipients. Lancet. 2003;361(9363):1099-1101.

57. Buxmann H, Hamprecht K, Meyer-Wittkopf M, Friese K. Primary human cytomegalovirus (HCMV) infection in pregnancy. Deutsches Ärzteblatt International. 2017;114(4):45-52.

58. Li C, Houser BL, Nicotra ML, Strominger JL. HLA-G homodimer-induced cytokine secretion through HLA-G receptors on human decidual macrophages and natural killer cells. Proc Natl Acad Sci U S A. 2009;106(14):5767-5772.

59. Arase H, Mocarski ES, Campbell AE, Hill AB, Lanier LL. Direct recognition of cytomegalovirus by activating and inhibitory NK cell receptors. Science. 2002;296(5571):1323-1326.

60. Sun JC, Beilke JN, Lanier LL. Adaptive immune features of natural killer cells. Nature. 2009;457(7229):557-561.

61. Aguilar OA, et al. A viral immunoevasin controls innate immunity by targeting the prototypical natural killer cell receptor family. Cell. 2017;169(1):58-71.e14.

62. Rölle A, Brodin P. Immune adaptation to environmental influence: the case of NK cells and HCMV. Trends Immunol. 2016;37(3):233-243.

63. Ljungman P, et al. Definitions of cytomegalovirus infection and disease in transplant patients for use in clinical trials. Clin Infect Dis. 2017;64(1):87-91.

64. Fu L, Hazes B, Burshtyn DN. The first Ig domain of KIR3DL1 contacts MHC class I at a secondary site. J Immunol. 2011;187(4):1816-1825.

65. Li NL, Fu L, Uchtenhagen H, Achour A, Burshtyn DN. Cis association of leukocyte Ig-like receptor 1 with MHC class I modulates accessibility to antibodies and HCMV UL18. Eur J Immunol. 2013;43(4):1042-1052.

66. Wagner CS, Rölle A, Cosman D, Ljunggren HG, Berndt KD, Achour A. Structural elements underlying the high binding affinity of human cytomegalovirus UL18 to leukocyte immunoglobulinlike receptor-1. J Mol Biol. 2007;373(3):695-705.

67. Emsley P, Lohkamp B, Scott WG, Cowtan K. Features and development of Coot. Acta Crystallogr D Biol Crystallogr. 2010;66(pt 4):486-501. 